\title{
A rolling horizon stochastic programming approach for the integrated planning of production and utility systems
}

\author{
Nur I. Zulkafli a,b and Georgios M. Kopanos ${ }^{a,{ }^{*}}$ \\ ${ }^{a}$ Cranfield University, School of Water, Energy and Environment, Bedfordshire MK43 OAL, \\ United Kingdom. \\ b Universiti Teknikal Malaysia Melaka, Centre of Advanced Research on Energy, Durian \\ Tunggal, Melaka 76100, Malaysia. \\ *Corresponding author: g.kopanos@cranfield.ac.uk
}

\begin{abstract}
This study focuses on the operational and resource-constrained condition-based cleaning planning problem of integrated production and utility systems under uncertainty. For the problem under consideration, a two-stage scenario-based stochastic programming model that follows a rolling horizon modelling representation is introduced; resulting in a hybrid reactiveproactive planning approach. In the stochastic programming model, all the binary variables related to the operational status (i.e., startup, operating, shutdown, under online or offline cleaning) of the production and utility units are considered as first-stage variables (i.e., scenario independent), and most of the remaining continuous variables are second-stage variables (i.e., scenario dependent). In addition, enhanced unit performance degradation and recovery models due to the cumulative operating level deviation and cumulative operating times are presented. Terminal constraints for minimum inventory levels for utilities and products as well as maximum unit performance degradation levels are also introduced. Two case studies are presented to highlight the applicability and the particular features of the proposed approach as an effective means of dealing with the sophisticated integrated planning problem considered in highly dynamic environments.
\end{abstract}

Keywords: production planning, cleaning, utility system, stochastic programming, rolling horizon, optimization.

\section{Introduction}

The process industry is a key economic sector globally. The global market share and business performance of the process industry is heavily based on the value that can be generated from its assets and while the range of valuable assets is large, nearly all the economic value in terms of operating profit in the process industry is a direct result of operations of plant equipment (Christofides et al., 2007). Also, major plant equipment constitute highly expensive capital assets that are typically subject to performance degradation and require periodic maintenance to avoid their damage or inefficient operation. Typically, maintenance planning follows very conservative approaches and is done separately from the production planning. Such approaches result in increased needs for maintenance resources (and associated costs), material waste, and productivity losses. All these, make clear the imperative need for systematic approaches for the efficient management of equipment operations and maintenance to preserve the major assets of a process industry and increase financial gains and competitiveness. 
In process industries, a sequential approach is typically used for the operational planning of utility and production systems. First, the planning of the production system is performed considering simply upper bounds on the availability of utilities. Once the production plan is derived, the utility needs of the production are known. This information is then used to obtain the operational planning of the utility system. This sequential approach provides suboptimal solutions (mainly in terms of resource and energy efficiency and costs) because the two interconnected systems are not optimized at the same time. Importantly, the sequential approach often faces the risk of providing utilities generation targets that cannot be met by the energy system (i.e., infeasible solutions), and in that case a re-planning of the production system is usually employed (Zulkafli and Kopanos, 2016). What is more, conservative maintenance planning is usually performed separately from operational planning which typically does not consider the dynamic condition of the equipment.

Modern process industries operate in highly dynamic environments that usually involve significant fluctuations on key operational, costs and market related parameters (e.g., demand fluctuations, prices variations or unit breakdowns). This makes essential the development and use of efficient planning approaches to deal with such types of uncertainties. There are two major types of planning approaches to deal with uncertainties, namely reactive and proactive approaches. In general, reactive approaches involve the repetitive solution of the deterministic planning problem within a rolling horizon framework, and are especially suitable for highly dynamic environments with limited information about the behavior of the uncertainty (Zulkafli and Kopanos, 2017). In simple words, these approaches basically rely on a wait and react approach with respect to unexpected events. Proactive approaches are typically used when some information about the behavior of the uncertainty is available, and their purpose is to provide solutions that will be immune to uncertainty. These approaches rely on an act before it happens basis. Stochastic programming or robust optimization are usually used in proactive planning approach. In general, proactive approaches propose more conservative solutions in comparison with reactive approaches, and are more suitable for less flexible environments in terms of changing frequently major operational decisions.

A number of works that proposed proactive approaches for operational planning problems can be found in the open literature. For example, Cobuloglu and Esra Büyüktahtakın (2017) proposed a two-stage stochastic programming model for maximizing economic and environmental aspects of food and biofuel production under yield and prices uncertainty. Choi et al. (2016) presented a stochastic programming model under a Monte-Carlo simulation to develop a multi-period energy planning model under uncertainty in market prices and demands for energy resources. Huang et al. (2016) presented a two-stage stochastic programming model for the electricity planning under demand uncertainty. Kostin et al. (2012) studied a multi-scenario problem on the design and planning of integrated bioethanol-sugar supply chains under demand uncertainty. Other works have developed proactive approaches for cleaning planning problems. For instance, Gössinger et al. (2017) presented a conditionbased cleaning policy to deal with stochastic deterioration processes. In the same line, Samuelson et al. (2017) presented a stochastic programming model for different cleaning strategies in continuously deteriorating systems. A two-stage nonlinear stochastic programming model for production and cleaning planning with yield and demand uncertainty was proposed by Ekin (2017), while Khatab et al. (2017) studied the cleaning planning problem for a multi-component system with stochastic durations of alternative cleaning actions. Zhou et al. (2016) presented an optimal cleaning policy of a parallel-series system considering stochastic and economic dependence under limited cleaning resources. The 
optimal cleaning schedule for heat exchanger network in an oil refinery under fouling and different aging scenarios was studied by Diaby et al. (2016) while Biyant et al. (2016) used different stochastic optimization methods developed for the optimal cleaning schedule in crude preheat trains. Among a limited number of works that combine reactive and proactive approaches, Silvente et al. (2015) developed a rolling horizon stochastic programming approach for the energy supply and demand management of microgrids. The authors further developed their model to consider a rolling horizon approach for optimal management of microgrid under stochastic uncertainty (Silvente et al., 2017). In addition, Gupta and Maranas (2000) studied a two-stage stochastic programming model to solve supply-chain planning problem under demand uncertainty through a rolling horizon framework.

In fact, most of the previous studies in literatures have addressed separately the operational planning problems or cleaning planning problems (i.e., sequential approach) under uncertainty for either utility or production system. A brief literature reviews on sequential approaches in process industries and a discussion on the need for integrated plant-wide planning approaches can be found in Zulkafli and Kopanos (2016). Importantly, Zulkafli and Kopanos (2017) showed that significant total cost reductions (from 5\% to $32 \%$ ) can be achieved if an integrated planning approach is used instead of the sequential alternative. Therefore, there is an important need for development of integrated planning approaches that also account efficiently for uncertainty to deal with the dynamic nature of the process industries.

This paper presents a two-stage scenario-based stochastic programming approach for the integrated planning of utility and production systems under uncertainty. It is assumed that some information about the behavior of the uncertainty parameters is known (i.e., number of scenarios with associated probability of occurrence, and given parameter values for each scenario). In particular, this study is a major extension of our previous work (Zulkafli and Kopanos, 2017) by: (i) providing a two-stage scenario-based stochastic programming version of a modification of the previously deterministic model, (ii) introducing an improved cumulative operating level deviation model for condition-based cleaning policies, (iii) defining improved terminal constraints for the maximum allowable unit performance degradation level (i.e., minimum performance level) at the end of the planning horizon, (iv) incorporating the resulting two-stage scenario-based stochastic programming model into a rolling horizon framework to readily deal with various types of uncertainties. The proposed approach follows a plant-wide condition-based approach for the cleaning actions that explicitly consider the condition of the units as a result of the optimized operational planning of the production and utility systems. To the best of our knowledge this is the first work that proposes a rolling horizon stochastic programming approach for the simultaneous operational and condition-based planning for integrated production and utility systems.

This paper is organized as follows. Section 2 provides a formal statement of the problem under study. The proposed optimization framework is presented in Section 3 followed by two case studies in Section 4. Finally, some concluding remarks with ongoing research directions are provided in Section 5.

\section{Problem Statement}

This work studies the stochastic version of the integrated operational and condition-based cleaning planning of production and utility systems under alternative resource-constrained cleaning policies, by considering performance degradation and recovery for utility and production units. Demand profiles for products are considered as the uncertain parameters of the problem in question, and it is assumed that they can be modeled by defining a number of 
different scenarios with given probability of occurrence. This results into a two-stage scenariobased stochastic programming planning problem which is formally defined in terms of following items:

- A given planning horizon divided into a number of equal-length time periods $t \in T$.

- A set of scenarios $n \in N$ with given probability of occurrence for each scenario $\delta_{n}^{p}$.

- A set of resources $e \in E$ that are divided into product $\left(e \in E^{P R}\right)$ and utilities $\left(e \in E^{U T}\right)$.

- Given demand profiles for products per scenario $\zeta_{(n, e, t)}$ (i.e., stochastic parameter).

- A set of units $i \in I$ that are classified to utility $\left(i \in U T_{i}\right)$ and production $\left(i \in P R_{i}\right)$ units and could produce a number of resources $e \in E_{i}$. Maximum (minimum) operating levels $\kappa_{(i, t)}^{\max }$ $\left(\kappa_{(i, t)}^{\min }\right)$ for utility units and production levels $\bar{\kappa}_{(i, e, t)}^{\max }\left(\bar{\kappa}_{(i, e, t)}^{\min }\right)$ for production units are given. For every unit that is subject to startup and shutdown actions $\left(i \in I^{S F}\right)$, the startup $\left(\phi_{(i, t)}^{S}\right)$ and shutdown $\left(\phi_{(i, t)}^{F}\right)$ costs are also given. For any unit that is subject to minimum runtime and shutdown time restrictions (i.e., $i \in I^{S-\min }$ and $i \in I^{F-\min }$, respectively), the minimum runtime after its last startup $\omega_{i}$ and the minimum idle time after its last shutdown $\psi_{i}$ are also defined.

- A set of resource-dedicated inventory tanks $z \in Z_{e}$ that can receive resources from units $i \in I_{z}^{+}$and send resources to units $i \in I_{z}^{-}$. Inventory tanks have a given maximum (minimum): inventory tank level $\beta_{(e, z)}^{\max }\left(\beta_{(e, z)}^{\min }\right)$, inlet resource flow $\beta_{(e, z, t)}^{+, \max }\left(\beta_{(e, z, t)}^{+, \min }\right)$, and outlet utility resource flow $\beta_{(e, z, t)}^{-, \max }\left(\beta_{(e, z, t)}^{-, \min }\right)$. Initial inventory tank levels $\tilde{\beta}_{(e, z)}$ and losses coefficients $\beta_{z}^{\text {loss }}$ are also given.

- Different cleaning policies for the units are considered. In particular, a unit could be subject to: (i) flexible time-window offline cleaning $\left(i \in F M_{i}\right)$ with a given earliest $\tau_{i}^{e s}$ and latest $\tau_{i}^{l s}$ starting time, (ii) in-progress offline cleaning carried over from the previous planning horizon $\left(i \in D M_{i}\right)$, or (iii) condition-based cleaning $\left(i \in C B_{i}\right)$ with known performance degradation rates. Two types of condition-based cleaning tasks are considered, namely: online cleaning tasks $\left(C B_{i}^{o n}\right)$ with given recovery factors $\rho_{i}^{\text {rec }}$, and offline cleaning tasks $\left(C B_{i}^{o f f}\right)$.

- A set of alternative cleaning tasks options $q \in Q_{i}$ for each unit that is subject to flexible time-window cleaning $\left(i \in F M_{i}\right)$ or offline condition-based cleaning $\left(i \in C B_{i}^{\text {off }}\right)$. The cleaning tasks options are characterized by different durations $v_{(i, q)}$, cleaning resource requirements $\vartheta_{(i, q)}^{\text {off }}$, and associated cleaning costs $\phi_{(i, q, t)}^{\text {off }}$.

- For every production unit $i \in I_{e}^{P R}$, fixed and variable utility needs for the production of products are given $\left(\bar{\alpha}_{\left(i, e, e^{\prime}\right)}\right.$ and $\alpha_{\left(i, e, e^{\prime}\right)}$, respectively).

- Given variable and fixed operating costs for production and utility units, $\phi_{(i, e, t)}^{P R, v \text {-var }}$ and $\phi_{(i, e, t)}^{P R, o p-f i x}$, and $\phi_{(i, t)}^{U T, o p-v a r}$ and $\phi_{(i, t)}^{U T, o p-f i x}$, respectively. 
- Given purchase prices for acquiring utilities and products from external sources, $\phi_{(e, i, t)}^{U T, e x}$ and $\phi_{(e, t)}^{P R, e x}$, respectively.

- A given time-varying energy price profile $\phi_{(i, t)}^{p w}$.

For the planning horizon considered, the optimization goal is to minimize the total cost which mainly includes unit operational and cleaning costs and resource purchases. In order to achieve this, for every time period, the key decisions to be optimized are: the operational status of each production and utility unit (i.e., startup, shutdown, in operation, idle, under online or offline cleaning); the selection of the timing and the offline cleaning task option for each unit; the operating level for each production and utility unit for each scenario; the inventory level for utilities and product resources for each scenario; and the utility requirements per scenario for each production unit.

The decision variables of the two-stage scenario-based stochastic programming problem under consideration are divided in first-stage and second-stage variables as shown below.

First-stage variables (i.e., scenario independent):

- $X_{(i, t)}=\left\{\begin{array}{l}1 \text { if unit } i \text { is operating during time period } t \\ 0 \text { otherwise. }\end{array}\right.$

- $S_{(i, t)}=\left\{\begin{array}{l}1 \text { if unit } i \text { starts up at the beginning of time period } t \\ 0 \text { otherwise. }\end{array}\right.$

- $F_{(i, t)}=\left\{\begin{array}{l}1 \text { if unit } i \text { shuts down at the beginning of time period } t \\ 0 \text { otherwise. }\end{array}\right.$

- $H_{(i, q, t)}=\left\{\begin{array}{l}1 \text { if cleaning task option } q \text { for } i \in\left(C B_{i}^{\text {off }} \cup F M_{i}\right) \text { begins at the start of time period } t \\ 0 \text { otherwise. }\end{array}\right.$

- $W_{(i, t)}=\left\{\begin{array}{l}1 \text { if an offline cleaning task for } i \in\left(C B_{i}^{\text {off }} \cup F M_{i}\right) \text { begins at the start of time period } t \\ 0 \text { otherwise. }\end{array}\right.$

- $V_{(i, t)}=\left\{\begin{array}{l}1 \text { if an online cleaning task for } i \in\left(C B_{i}^{o n} \cap U T_{i}\right) \text { takes place in time period } t \\ 0 \text { otherwise. }\end{array}\right.$

- $V_{(i, e, t)}^{P R}=\left\{\begin{array}{l}1 \text { if an online cleaning task for } i \in\left(C B_{i}^{o n} \cap P R_{i}\right) \text { that produces } e \in E_{i} \text { in time period } t \\ 0 \text { otherwise. }\end{array}\right.$

- $Y_{(i, e, t)}=\left\{\begin{array}{l}1 \text { if production unit } i \in P R_{i} \text { produces product } e \text { during time period } t, \\ 0 \text { otherwise. }\end{array}\right.$

- $R_{(i, t)}$ : cumulative time of operation for units subject to condition-based cleaning.

Second-stage variables (i.e., scenario dependent):

- Operating levels for utility units $\bar{Q}_{(n, i, t)}$.

- Production levels for utilities and products from their respective unit $Q_{(n, i, e, t)}$.

- Inventory levels for utilities and products $B_{(n, e, z, t)}$. 
- Total inlet flow of utilities and products to their respective inventory tanks $B_{(n, e, z, t)}^{+}$.

- Total outlet flow of utilities and products from their respective inventory tanks $B_{(n, e, z, t)}^{-}$.

- Extra energy consumption of units due to their performance degradation $U_{(n, i, t)}$.

- Cumulative operating level deviation for units subject to condition-based cleaning $D_{(n, i, t)}$.

- Operating level deviations of production units from their reference operating level $Q_{(n, i, e, t)}^{d e v}$.

- Operating level deviations of utility units from their reference operating level $\bar{Q}_{(n, i, t)}^{d e v}$.

- Purchases of utilities $N S_{(n, e, i, t)}^{U T}$ or products $N S_{(n, e, t)}^{F P}$. 


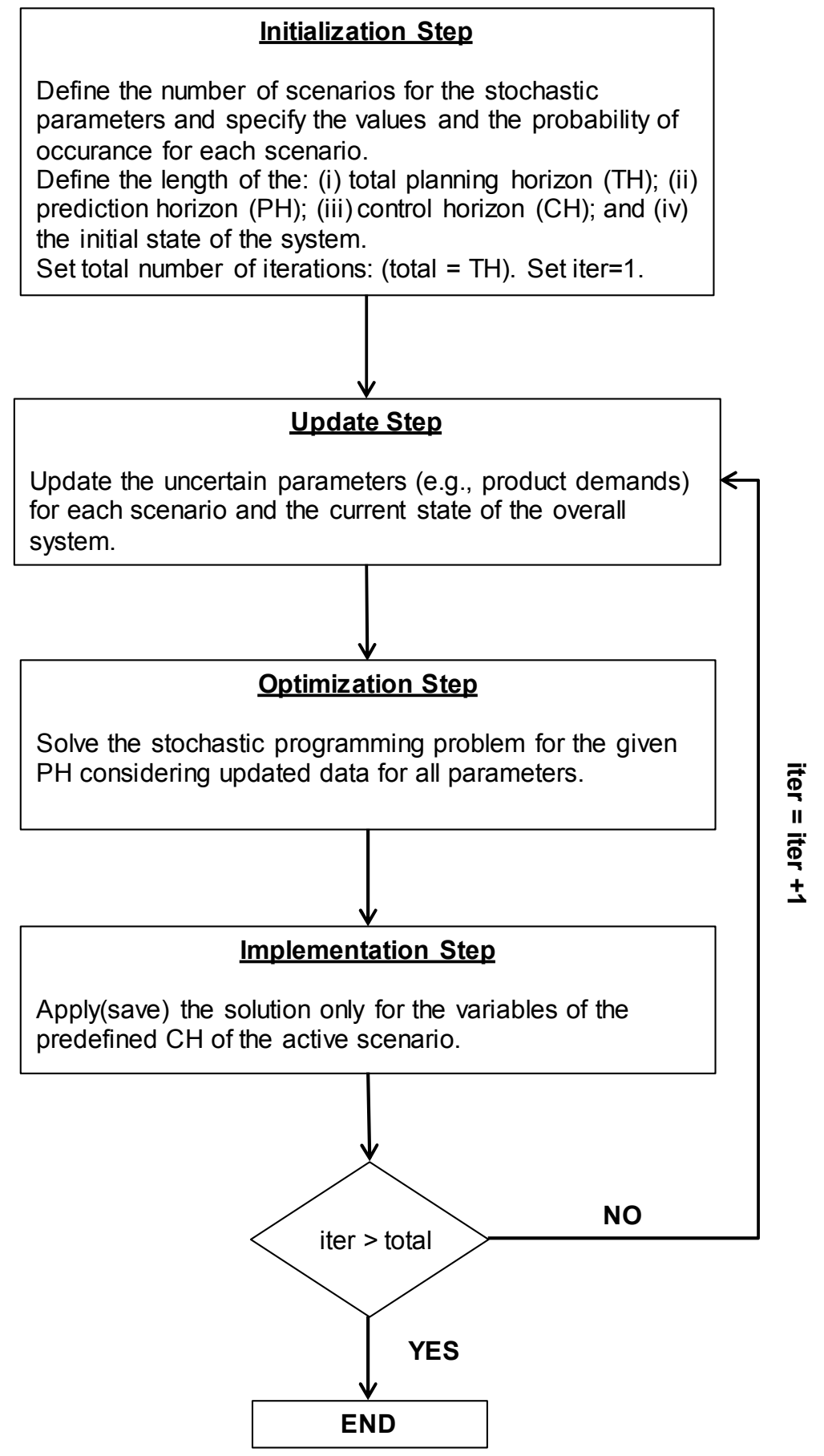

Figure 1: Planning via a rolling horizon stochastic programming method.

\section{Optimization Framework}

This part presents the proposed stochastic programming model for the integrated planning problem described in the previous section. This stochastic programming model follows a rolling-horizon modelling representation, and that way results in a hybrid reactive-proactive planning approach, when applied within a rolling-horizon scheme. Figure 1 shows a schematic representation of the steps of the proposed planning approach that work as follows. First, one needs to define a number of scenarios with assigned probabilities of occurrence and specified values for the uncertain parameters considered. Next, a prediction horizon is defined for which 
the stochastic programming model is solved. The length of the prediction horizon depends on the quality of the available information of the uncertain parameters. In the rolling-horizon approach, it is implemented in practice the solution of a limited number of periods (i.e., usually just that of the first time period of the prediction horizon) that have been considered in the prediction horizon. In such approaches, it is essential to update properly the initial state of the overall system before solving the optimization problem for the given prediction horizon. Especially, if a scenario-based stochastic approach is used, the active scenario (i.e., scenario that eventually occurred) should be known/defined and update the initial state of the system with respect to this active scenario. In this study, the main parameters that describe the initial state of the overall system are: (i) the inventory levels for utilities and products; (ii) the cumulative time of operation for each unit; (iii) the cumulative operating level deviation for each unit; (iv) the current operating status of each unit; (v) the startup and shutdown history of each unit; (vi) the online and offline cleaning history of each unit; (vii) the cleaning resources history of units; and (viii) the demands for products per scenario considered. A more detailed description and discussion on the reactive planning via a rolling horizon framework can be found in Zulkafli and Kopanos (2017).

The stochastic programming model presented is an enhanced modified version of the deterministic model of our previous work (Zulkafli and Kopanos, 2017). For this reason, constraints that remain unchanged from its deterministic version, proper references will be given to the constraints of our previous work to avoid unnecessary repetitions. A description of the proposed optimization framework follows.

\subsection{Major operational and cleaning decisions.}

Constraints related to major operational and cleaning decisions are modeled through firststage binary variables. These constraints are the same with those of the deterministic version of the model presented in our previous work. More specifically, the stochastic programming model includes constraints (1) to (9) and (23) to (25) from Zulkafli and Kopanos (2017). In brief, these constraints model the operational status for units with respect to startup, operation, shutdown, and online or offline cleaning decisions as given in the Supplementary Material. These constraints model: (i) minimum run and shutdown periods (i.e., SM1); (ii) inprogress offline cleaning tasks (i.e., SM2.1); (iii) flexible time-window offline cleaning tasks(i.e., SM2.2); (iv) condition-based online cleaning tasks (i.e., SM2.3); (v) operational constraints for offline cleaning tasks(i.e., SM2.4); and (vi) resource limitations for cleaning resources(i.e., SM2.5). A detailed description of these constraints can be found in Zulkafli and Kopanos (2017).

\subsubsection{Performance degradation and recovery models for units.}

For each scenario, the performance of any unit that is subject to condition-based cleaning is modeled through the extra energy consumption $U_{(n, i, t)}$ due to its deviation from its completely clean condition. It is assumed that the performance of a unit decreases as this extra energy consumption increases. To avoid the energy inefficient use and potential damage of the unit, this extra energy consumption for the units under operation should not exceed a maximum extra energy consumption limit $v_{i}^{\max }$, as defined by:

$U_{(n, i, t)} \leq v_{i}^{\max } X_{(i, t)} \quad \forall n \in N, \forall i \in C B_{i}, \forall t \in T$ 
The extra energy consumption of an operating unit is related to its cumulative time of operation $R_{(i, t)}$ and its cumulative operating level deviation $D_{(n, i, t)}$, through parameters $\delta_{i}$ and $\delta_{i}^{q}$ that represent the corresponding degradation rates, as given by:

$$
\begin{array}{lr}
U_{(n, i, t)} \geq \delta_{i} R_{(i, t)}+\delta_{i}^{q} D_{(n, i, t)}-v_{i}^{\max }\left(1-X_{(i, t)}\right) & \forall n \in N, \forall i \in C B_{i}, \forall t \in T \\
U_{(n, i, t)} \leq \delta_{i} R_{(i, t)}+\delta_{i}^{q} D_{(n, i, t)}+v_{i}^{\max }\left(1-X_{(i, t)}\right) & \forall n \in N, \forall i \in C B_{i}, \forall t \in T
\end{array}
$$

Please refer to Zulkafli and Kopanos (2017) for a more detailed discussion on the proposed performance degradation and recover models.

Cumulative time of operation. The variables that describe the cumulative time of operation are first-stage variables, and the corresponding constraints considered are the same with the deterministic constraints (12) to (15) of our previous work (Zulkafli and Kopanos, 2017).

Cumulative operating level deviation. The variables that describe the cumulative operating level deviation are second-stage variables, and the corresponding constraints are presented here. First, similarly to the cumulative time of operation, the occurrence of an offline cleaning task in a unit resets its cumulative operating level deviation to zero, as defined by:

$$
D_{(n, i, t)} \leq \mu_{(i, t)}\left(1-W_{(i, t)}\right) \quad \forall n \in N, \forall i \in C B_{i}^{o f f}, \forall t \in T
$$

Parameters $\mu_{(i, t)}$ are sufficient big numbers that could be calculated through the corresponding maximum extra energy consumption and degradation rate parameters.

In comparison with our previous work, in this study we present improved sets of constraints for the modeling of the cumulative operating level deviation for units subject to condition-based cleaning. More specifically, in this study the cumulative operating level deviation of a unit resets to zero only after the occurrence of an offline cleaning task while in our previous it was assumed that this happens after the shutdown of the unit.

The new sets of constraints for the modeling of the cumulative operating level deviation of utility units subject to condition-based cleaning are presented below:

$$
\begin{aligned}
& \bar{Q}_{(n, i, t)}^{d e v} \leq\left(\frac{\left|q_{(i, t)}^{r e f}-\bar{Q}_{(n, i, t)}\right|}{q_{(i, t)}^{r e f}}\right)+\mu_{(i, t)}\left(1-X_{(i, t)}\right) \quad \forall n \in N, \forall i \in\left(C B_{i} \cap U T_{i}\right), t \in T \\
& \bar{Q}_{(n, i, t)}^{d e v} \geq\left(\frac{\left.\left|q_{(i, t)}^{r e f}-\bar{Q}_{(n, i, t)}\right|\right)}{q_{(i, t)}^{r e f}}\right)-\mu_{(i, t)}\left(1-X_{(i, t)}\right) \quad \forall n \in N, \forall i \in\left(C B_{i} \cap U T_{i}\right), t \in T \\
& \bar{Q}_{(n, i, t)}^{d e v} \leq \mu_{(i, t)} X_{(i, t)} \quad \forall n \in N, \forall i \in\left(C B_{i} \cap U T_{i}\right), t \in T \\
& D_{(n, i, t)} \leq \tilde{\rho}_{i}^{q}+\bar{Q}_{(n, i, t)}^{d e v}+\mu_{(i, t)}\left(W_{(i, t)}+V_{(i, t)}\right) \quad \forall n \in N, \forall i \in\left(C B_{i} \cap U T_{i}\right), t \in T: t=1 \\
& D_{(n, i, t)} \leq \mathrm{D}_{(n, i, t-1)}+\bar{Q}_{(n, i, t)}^{d e v}+\mu_{(i, t)}\left(W_{(i, t)}+V_{(i, t)}\right) \quad \forall n \in N, \forall i \in\left(C B_{i} \cap U T_{i}\right), t \in T: t>1 \\
& D_{(n, i, t)} \geq \tilde{\rho}_{i}^{q}+\bar{Q}_{(n, i, t)}^{d e v}-\mu_{(i, t)}\left(W_{(i, t)}+V_{(i, t)}\right) \quad \forall n \in N, \forall i \in\left(C B_{i} \cap U T_{i}\right), t \in T: t=1 \\
& D_{(n, i, t)} \geq \mathrm{D}_{(n, i, t-1)}+\bar{Q}_{(n, i, t)}^{d e v}-\mu_{(i, t)}\left(W_{(i, t)}+V_{(i, t)}\right) \quad \forall n \in N, \forall i \in\left(C B_{i} \cap U T_{i}\right), t \in T: t>1 \\
& D_{(n, i, t)} \geq\left(\tilde{\rho}_{i}^{q}+\bar{Q}_{(n, i, t)}^{d e v}\right)\left(1-\rho_{i}^{r e c}\right)-\mu_{(i, t)}\left(1-V_{(i, t)}\right) \quad \forall n \in N, \forall i \in\left(C B_{i}^{o n} \cap U T_{i}\right), t \in T: t=1 \\
& D_{(n, i, t)} \geq\left(\mathrm{D}_{(i, t-1)}+\bar{Q}_{(n, i, t)}^{d e v}\right)\left(1-\rho_{i}^{r e c}\right)-\mu_{(i, t)}\left(1-V_{(i, t)}\right) \quad \forall n \in N, \forall i \in\left(C B_{i}^{o n} \cap U T_{i}\right), t \in T: t>1
\end{aligned}
$$


New variables $\bar{Q}_{(n, i, t)}^{d e v}$ have been defined to describe the additional cumulative operating level deviation at each time period from a reference operating level $q_{(i, t)}^{r e f}$. That way the cumulative operating level deviation variables $D_{(n, i, t)}$ do not reset to zero whenever a unit shuts down (i.e., if $X_{(i, t)}=0$, then $\bar{Q}_{(n, i, t)}^{d e v}=0$ and $\left.D_{(n, i, t)}=D_{(n, i, t-1)}\right)$. The cumulative operating level deviation can be reset to zero if and only if a utility unit undergoes offline cleaning. Under online cleaning periods, the cumulative operating level deviation of a utility unit is reduced partially by a given recovery factor, as defined by constraints (7).

In the same line, the cumulative operating level deviation of production units subject to condition-based cleaning is modeled by the new sets of constraints presented below:

$$
\begin{array}{ll}
Q_{(n, i, e, t)}^{d e v} \leq\left(\frac{\mid q_{(i, e, t)}^{r e f}-Q_{(n, i, e, t)}}{q_{(i, e, t)}^{r e f}}\right)+v_{i}^{\max }\left(1-Y_{(i, e, t)}\right) \quad \forall n \in N, \forall i \in\left(C B_{i} \cap P R_{i}\right), e \in E_{i}, t \in T \\
Q_{(n, i, e, t)}^{d e v} \geq\left(\frac{\left.\left|q_{(i, e, t)}^{r e f}-\bar{Q}_{(n, i, e, t)}\right|\right)}{q_{(i, e, t)}^{r e f}}\right)-v_{i}^{\max }\left(1-Y_{(i, e, t)}\right) \quad \forall n \in N, \forall i \in\left(C B_{i} \cap P R_{i}\right), e \in E_{i}, t \in T \\
Q_{(n, i, e, t)}^{d e v} \leq \mu_{(i, t)} Y_{(i, e, t)} \quad \forall n \in N, \forall i \in\left(C B_{i} \cap P R_{i}\right), e \in E_{i}, t \in T \\
D_{(n, i, t)} \leq \tilde{\rho}_{i}^{q}+\sum_{e \in E_{i}} Q_{(n, i, e, t)}^{d e v}+\mu_{(i, t)}\left(W_{(i, t)}+V_{(i, t)}\right) \\
D_{(n, i, t)} \leq \mathrm{D}_{(i, t-1)}+\sum_{e \in E_{i}} Q_{(n, i, e, t)}^{d e v}+\mu_{(i, t)}\left(W_{(i, t)}+V_{(i, t)}\right) & \forall n \in N, \forall i \in\left(C B_{i} \cap P R_{i}\right), t \in T: t=1 \\
D_{(n, i, t)} \geq \tilde{\rho}_{i}^{q}+\sum_{e \in E_{i}} Q_{(n, i, e, t)}^{d e v}-\mu_{(i, t)}\left(W_{(i, t)}+V_{(i, t)}\right) & \forall n \in N, \forall i \in\left(C B_{i} \cap P R_{i}\right), t \in T: t>1 \\
D_{(n, i, t)} \geq \mathrm{D}_{(i, t-1)}+\sum_{e \in E_{i}} Q_{(n, i, e, t)}^{d e v}-\mu_{(i, t)}\left(W_{(i, t)}+V_{(i, t)}\right) & \forall n \in N, \forall i \in\left(C B_{i} \cap P R_{i}\right), t \in T: t>1 \\
D_{(n, i, t)} \geq\left(\tilde{\rho}_{i}^{q}+\sum_{e \in E_{i}} Q_{(n, i, e, t)}^{d e v}\right)\left(1-\rho_{i}^{r e c}\right)-\mu_{(i, t)}\left(1-V_{(i, t)}\right) & \forall n \in N, \forall i \in\left(C B_{i}^{o n} \cap P R_{i}\right), t \in T: t=1 \\
D_{(n, i, t)} \geq\left(\mathrm{D}_{(i, t-1)}+\sum_{e \in E_{i}} Q_{(n, i, e, t)}^{d e v}\right)\left(1-\rho_{i}^{r e c}\right)-\mu_{(i, t)}\left(1-V_{(i, t)}\right) & \forall n \in N, \forall i \in\left(C B_{i}^{o n} \cap P R_{i}\right), t \in T: t>1
\end{array}
$$

For every unit, parameter $\tilde{\rho}_{i}^{q}$ represents its cumulative operating level deviation just before the beginning of the planning horizon of interest (i.e., initial state).

\subsection{Utility and production units: operating levels bounds.}

Utility system. The utility system consists of a number of utility units that could generate a number of utility resources required by the production system. The operating level for each operating utility unit per scenario should be between its lower and upper operating level bounds $\left(\kappa_{(i, t)}^{\min }\right.$ and $\left.\kappa_{(i, t)}^{\max }\right)$. The maximum operating levels during online cleaning periods are modeled as discussed in our previous work (Zulkafli and Kopanos, 2017). The operating bounds are given by:

$\kappa_{(i, t)}^{\min } X_{(i, t)} \leq \bar{Q}_{(n, i, t)} \leq \kappa_{(i, t)}^{\max }\left(X_{(i, t)}-\pi_{i}^{o n} V_{(i, t)}\right) \quad \forall n \in N, \forall i \in\left(U T_{i} \cap C B_{i}^{o n}\right), t \in T$ 
Some types of utility units, such as combined heat and power units, generate at the same time more than one utility resources. The generated amount of any utility resource from each utility unit per scenario and time period is modeled by:

$$
Q_{(n, i, e, t)}=\rho_{(i, e)} \bar{Q}_{(n, i, t)} \quad \forall n \in N, \forall i \in U T_{i}, e \in E_{i}, t \in T
$$

Parameters $\rho_{(i, e)}$ denote the stoichiometry coefficients that relate the operating level of the utility unit with the generated amount of each utility resource type $\left(Q_{(n, i, e, t)}\right)$ that is cogenerated by the same utility system (e.g., heat to power ratio of a combined heat and power unit).

Production system. This study considers a single-stage production process with a number of different units operating in parallel for producing the whole set of desired products. Similarly to utility units, changes in the maximum production levels during online cleaning periods are considered. Therefore, the production bounds of this general case are given by:

$\bar{\kappa}_{(i, e, t)}^{\min } Y_{(i, e, t)} \leq Q_{(n, i, e, t)} \leq \bar{\kappa}_{(i, e, t)}^{\max }\left(Y_{(i, e, t)}-\pi_{i}^{o n} V_{(i, e, t)}^{P R}\right) \quad \forall n \in N, \forall i \in\left(P R_{i} \cap C B_{i}^{o n}\right), e \in E_{i}, t \in T$

The production unit could produce at most one product resource per time period as modeled by constraints (29) and (30) of our previous work (Zulkafli and Kopanos, 2017).

\subsection{Inventory tanks for utilities and products.}

The overall system contains a number of resource-dedicated inventory tanks for the storage of utilities and products. Decisions related to inventories depend on each scenario, and thus they are described by second-stage variables through the following set of constraints.

$$
\begin{aligned}
& B_{(n, e, z, t)}^{+}=\sum_{i \in\left(I_{e} \cap I_{z}^{+}\right)} Q_{(n, i, e, t)} \quad \forall n \in N, e \in E, z \in Z_{e}, t \in T \\
& \varepsilon_{(e, z, t)}^{+, m i n} \leq B_{(n, e, z, t)}^{+} \leq \varepsilon_{(e, z, t)}^{+, \max } \quad \forall n \in N, e \in E, z \in Z_{e}, t \in T \\
& B_{(n, e, z, t)}=\tilde{\beta}_{(e, z)}+B_{(n, e, z, t)}^{+}-B_{(n, e, z, t)}^{-} \quad \forall n \in N, e \in E, z \in Z_{e}, t \in T: t=1 \\
& B_{(n, e, z, t)}=\left(1-\beta_{z}^{l o s s}\right) B_{(n, e, z, t-1)}+B_{(n, e, z, t)}^{+}-B_{(n, e, z, t)}^{-} \quad \forall n \in N, e \in E, z \in Z_{e}, t \in T: t>1 \\
& \xi_{(e, z)}^{\min } \leq B_{(n, e, z, t)} \leq \xi_{(e, z)}^{\max } \quad \forall n \in N, e \in E, z \in Z_{e}, t \in T
\end{aligned}
$$

Constraints (15) define the total inlet flow $\left(B_{(n, e, z, t)}^{+}\right)$to each inventory tank from units $I_{z}^{+}$that are connected to. Constraints (16) give the lower and upper bounds on these inlet flows. Resource balances for every inventory tank, scenario and time period are modeled by constraints (17), where variables $B_{(n, e, z, t)}$ indicate the inventory level per scenario, resource and inventory tank at the end of each time period and variables $B_{(n, e, z, t)}^{-}$represent the outlet flow from each inventory tank per scenario. Parameters $\tilde{\beta}_{(e, z)}$ define the initial inventory for inventory tank at the beginning of the planning horizon (i.e., initial state) and parameters $\beta_{z}^{\text {loss }}$ give the losses coefficients. Inventory levels bounds are defined by constraints (18).

For each time period and scenario, the amount of each utility that leaves its dedicated inventory tank per scenario is equal to the total amount of utility consumed by the associated production units $I_{z}^{-}$. These outlet utility flows are bounded within a lower and upper limit. 


$$
\begin{aligned}
& B_{(n, e, z, t)}^{-}=\sum_{i \in\left(P R_{i} \cap I_{z}^{-}\right)} B_{(n, e, z, i, t)}^{U T,-} \quad \forall n \in N, e \in E^{U T}, z \in Z_{e}, t \in T \\
& \varepsilon_{(e, z, t)}^{-, \min } \leq B_{(n, e, z, t)}^{-} \leq \varepsilon_{(e, z, t)}^{-, \max } \quad \forall n \in N, \forall e \in E^{U T}, z \in Z_{e}, t \in T
\end{aligned}
$$

\subsection{Demands for products.}

For every scenario and time period, demands for products need to be satisfied, according to:

$$
N S_{(n, e, t)}^{F P}+\sum_{z \in Z_{e}} B_{(n, e, z, t)}^{-}=\zeta_{(n, e, t)} \quad \forall n \in N, e \in E^{P R}, t \in T
$$

Variables $N S_{(n, e, t)}^{F P}$ denote the unsatisfied product demand from the internal production system. If the demands for products cannot be met from the internal production system and there are no available external sources for product purchases, these variables represent lost sales of products. A high penalty cost is used in the objective function to avoid satisfying the demands for products from external sources.

\subsection{Requirements for utilities (link between utility and production systems).}

Utilities requirements provide the linking constraints between utility and production systems. For each time period and scenario, the utilities needs per production unit $I_{e}^{P R}$ consist of: (i) scenario-independent fixed utilities requirements that depend on the operational status of the production unit (first-stage variables); and (ii) scenario-dependent variable utilities requirements that depend on the production level of the production unit (second-stage variables). The utilities balance is then given by the following constraints:

$$
N S_{(n, e, i, t)}^{U T}+\sum_{z \in\left(Z_{e} \cap Z_{i}\right)} B_{(n, e, z, i, t)}^{U T,-}=\sum_{e^{\prime} \in\left(E^{P R} \cap E_{i}\right)}\left(\alpha_{\left(i, e, e^{\prime}\right)} Q_{\left(n, i, e^{\prime}, t\right)}+\bar{\alpha}_{\left(i, e, e^{\prime}\right)} Y_{\left(i, e^{\prime}, t\right)}\right) \quad \forall n \in N, e \in E^{U T}, i \in I_{e}^{P R}, t \in T
$$

Variables $N S_{(n, e, i, t)}^{U T}$ represent the unsatisfied utility requirements. Similarly to the unsatisfied demand for products, high penalty costs for acquiring utilities from external sources are introduced in the objective function of the optimization problem to favor the generation of utilities from the internal utility system.

\subsection{Objective function}

The optimization goal is to minimize the total cost of the production and the utility system along with the purchases of products and utilities from external sources. More specifically, the objective function includes: startup and shutdown costs for units, total cleaning costs related to online and offline cleaning tasks of production and utility units that are subject to performance degradation variable, variable and fixed operating costs for units, penalty or purchase costs for acquiring products or utilities from external sources, and total extra energy consumption costs for utility and production units that are subject to performance degradation modeling. The objective function considered in this study is then given by: 


$$
\min \left[\begin{array}{l}
\sum_{t \in T} \sum_{i \in I^{S F}}\left(\phi_{(i, t)}^{S} S_{(i, t)}+\phi_{(i, t)}^{F} F_{(i, t)}\right)+\sum_{t \in T} \sum_{i \in I^{U T}}\left(\phi_{(i, t)}^{U T, o p-f i x} X_{(i, t)}\right)+\sum_{t \in T} \sum_{i \in P R_{i}} \sum_{e \in E_{i}}\left(\phi_{(i, e, t)}^{P R, o p-f i x} Y_{(i, e, t)}\right) \\
+\sum_{t \in T}\left(\sum_{i \in C B_{i}^{o n}} \phi_{(i, t)}^{o n} V_{(i, t)}+\sum_{i \in\left(C B_{i}^{o f f} \cup F M_{i}\right)} \sum_{q \in Q_{i}} \phi_{(i, q, t)}^{o f f} H_{(i, q, t)}\right) \\
+\sum_{n \in N} \sum_{t \in T} \sum_{i \in I^{U T}}\left(\delta_{n}^{p} \phi_{(i, t)}^{U T, o p-v a r} \bar{Q}_{(n, i, t)}\right)+\sum_{n \in N} \sum_{t \in T} \sum_{i \in P R_{i}} \sum_{e \in E_{i}}\left(\delta_{n}^{p} \phi_{(i, e, t)}^{P R, o p-v a r} Q_{(n, i, e, t)}\right) \\
+\sum_{n \in N} \sum_{t \in T} \sum_{e \in E^{P R}}\left(\delta_{n}^{p} \phi_{(e, t)}^{P R, e x} N S_{(n, e, t)}^{F P}\right)+\sum_{n \in N} \sum_{t \in T} \sum_{e \in E^{U T}} \sum_{i \in I_{P R}^{e}}\left(\delta_{n}^{p} \phi_{(e, i, t)}^{U T, e x} N S_{(n, e, i, t)}^{U T}\right) \\
+\sum_{n \in N} \sum_{t \in T} \sum_{i \in C B_{i}}\left(\delta_{n}^{p} \phi_{(i, t)}^{p w} U_{(n, i, t)}\right)
\end{array}\right]
$$

In the above expression, the small-letter symbols correspond to the cost coefficients of the corresponding optimization variables. Probabilities of occurrence for each scenario are defined and multiplied with the associated second-stage variables. A detailed definition of each set, parameter, variable of the optimization framework can be found in the Nomenclature.

\subsection{Terminal constraints}

Terminal constraints are defined for the last time period of a given optimization problem as a means of preserving the operability and stability of the system at the end of the planning horizon considered. Here, we define terminal constraints for: the minimum inventory levels for utilities and products $\left(\lambda_{(e, z)}^{B}\right)$, and the maximum allowable unit performance degradation levels $\left(\lambda_{i}^{U}\right)$ for utility and production units, according to:

$$
\begin{array}{ll}
B_{(n, e, z, t)} \geq \lambda_{(e, z)}^{B} \xi_{(e, z)}^{\max } & \forall n \in N, e \in E, z \in Z_{e}, t \in T: t=|T| \\
\delta_{i} R_{(i, t)}+\delta_{i}^{q} D_{(n, i, t)} \leq \lambda_{i}^{U} v_{i}^{\max } & \forall n \in N, i \in C B_{i}, t \in T: t=|T|
\end{array}
$$

These terminal constraints are applied to any stochastic programming problem solved in this study.

\section{Case Studies}

In this part, two case studies are presented for the integrated planning of utility and production systems by employing the proposed stochastic programming approach. Both case studies follow the same plant layout that is displayed in Figure 2. The first case study considers a flexible time-window cleaning policy for production units and a condition-based cleaning policy for utility units. We consider alternative offline cleaning tasks options with respect to duration, cost and cleaning resource requirements. A maximum cleaning resources availability per time period is also considered. It is assumed that the reference operating level for any unit is equal to its maximum operating level. The second case study deals with the reactive planning using the proposed stochastic programming model through a rolling horizon framework. This problem considers a conditioned-based cleaning policy for both utility and production units. The resulting optimization problems have been solved in GAMS/CPLEX 12.6 in an Intel(R) core(TM) i7 under standard configurations. A 1\% optimality gap has been achieved for the first case study after $12 \mathrm{CPU} \mathrm{h}$ and a zero optimality gap for all optimization problems of the second case study. 


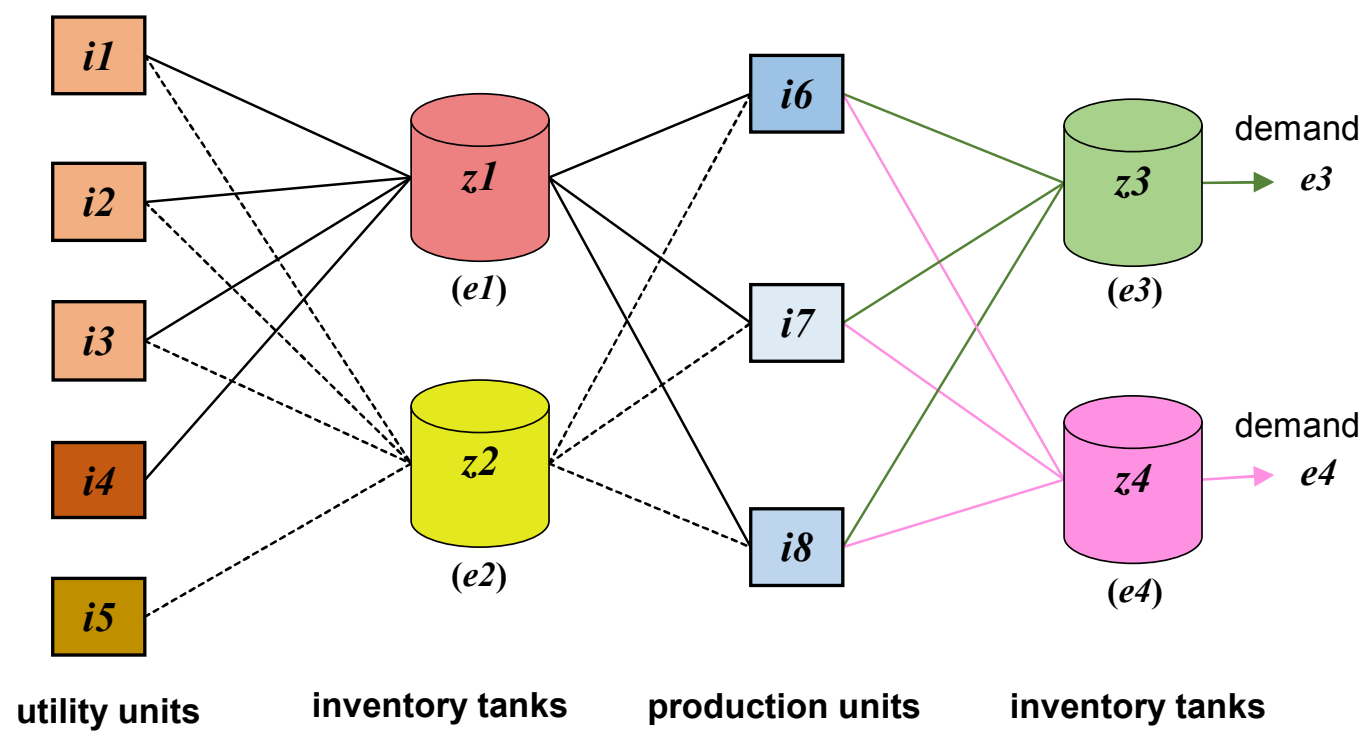

Figure 2: Plant layout for both case studies (utility and product flows from left to right).

\subsection{Case Study 1: Integrated Planning of Utility and Production Systems via Stochastic Programming}

In this case study, a combination of cleaning policies for units is studied. More specifically, flexible time-window offline cleaning tasks for production units and conditioned-based cleaning tasks (online and offline) for utility units are considered. The problem has been solved by the proposed two-stage scenario-based stochastic programming model.

\subsubsection{Case Study 1: Description.}

The production facility under consideration consists of five utility units $(i 1-i 5)$ and three production units $(i 6-i 8)$. Utility units could produce two utilities $(e 1, e 2)$ which could be either stored in their associated inventory tanks $(z 1, z 2)$ or consumed directly by the production units. Two products $(e 3, e 4)$ could be produced by the production units that can be either stored in their dedicated inventory tanks $(z 3, z 4)$ or meet directly the demands for products. A total planning horizon of 14 days (i.e., 2 weeks), divided in day time periods, is considered. Utility units are subject to online or offline conditioned-based cleaning, while production units are subject to flexible time-window offline cleaning. Earliest and latest starting cleaning times for all production units are on day 1 and 9. All parameters related to online and offline conditioned-based cleaning for utility units can be found in Table 6 of Zulkafli and Kopanos (2017). The only difference is the value for minimum time between two consecutive online cleanings $\left(\gamma_{i}^{o n}\right)$ that in this case study is considered to be equal to five time periods (i.e., four periods without online cleaning between two online cleanings). All parameters values that fully define the initial state of the overall system are given in Table 1. In this case study, initial parameters related to condition-based cleaning tasks (i.e., initial cumulative time of operation $\tilde{\rho}_{i}$ and initial state of unit with respect to its last online cleaning $\tilde{\gamma}_{i}^{o n}$ ) for production units are ignored, since in this problem instance we do not consider a condition-based cleaning policy for production units. 
Table 1. Initial state for utility and production units.

\begin{tabular}{lllllllll}
\hline Parameter & $i 1$ & $i 2$ & $i 3$ & $i 4$ & $i 5$ & $i 6$ & $i 7$ & $i 8$ \\
\hline$\tilde{\rho}_{i}$ & 2 & 2 & 7 & 9 & 10 & 6 & 7 & 3 \\
$\tilde{\gamma}_{i}^{o n}$ & 5 & 14 & 12 & 4 & 17 & 20 & 14 & 14 \\
$\tilde{\omega}_{i}$ & 2 & 16 & 7 & 1 & 7 & 7 & 5 & 18 \\
$\tilde{\psi}_{i}$ & 0 & 0 & 0 & 0 & 0 & 0 & 0 & 0 \\
$\tilde{\rho}_{i}^{q}$ & 2 & 3 & 4 & 3 & 1 & 4 & 3 & 1 \\
$\tilde{\chi}_{i}$ & 1 & 1 & 1 & 1 & 1 & 1 & 1 & 1 \\
\hline$\tilde{\beta}_{(e 1, z 1)}$ & 10 & units & & Initial inventory for utility $e 1$ & & \\
$\tilde{\beta}_{(e 2, z 2)}$ & 20 & units & & Initial inventory for utility $e 2$ & & \\
$\tilde{\beta}_{(e 3, z 3)}$ & 50 & units & Initial inventory for product $e 3$ & & \\
$\tilde{\beta}_{(e 4, z 4)}$ & 300 & units & Initial inventory for product $e 4$ & & \\
\hline
\end{tabular}

The following terminal constraints are imposed at the end of the planning horizon. The inventory levels for each inventory tank should be greater or equal to $10 \%$ from its corresponding maximum inventory level $\left(\xi_{(e, z)}^{\max }\right)$, and the performance degradation level of any utility unit should be lower or equal to $25 \%$ of the corresponding extra power consumption limit $\left(v_{i}^{\max }\right)$. Maximum total cleaning resources availability is 12 units for each time period. There are three alternative offline cleaning options $(q 1, q 2, q 3)$ that are characterized by different durations, cleaning resources requirements and associated costs. The cleaning duration $\left(v_{(i, q)}\right)$ for offline cleaning task options $q 1, q 2$ and $q 3$ is 3,4 and 5 days, respectively. The resource requirements $\left(\vartheta_{(i, q)}^{\text {off }}\right)$ for offline cleaning task options $q 1, q 2$ and $q 3$ is 6,4 and 3 cleaning resources, respectively. The resource requirement for online cleanings $\left(\vartheta_{i}^{\text {on }}\right)$ is 1 cleaning resource. A more detailed description of this case study and other main parameters can be found in Section 4.2.1 and Tables 1 to 5 of our previous work (Zulkafli and Kopanos, 2017).

For the stochastic programming problem, three different scenarios with respect to the demand profiles for products are considered, as displayed in Figure 3. More specifically, scenario $n 1$ represents medium demand profiles while scenario $n 2$ and $n 3$ correspond to high and low demand profiles, respectively. The probability of occurrence $\left(\delta_{n}^{p}\right)$ is equal to $30 \%$ for scenario $n 1,40 \%$ for scenario $n 2$, and $30 \%$ for scenario $n 3$. Figure 3 displays the normalized demand profiles for products by having as a reference the peak demand values of the high-demand scenario $n 2$. The major assumption in this work is that the three scenarios of demand profiles with respect to low, medium and high demand scenarios are considered the same for all time periods of the proposed two-stage stochastic programming model. Notice that, the number of scenarios considered may not be the most realistic scenarios in a real problem. The most appropriate method to deal with the real problem is multistage stochastic programming model (refer section 4.3). However, these three scenarios of demand profiles 
are sufficient to show the representation of the two-stage stochastic programming model in order to solve the problem under dynamic demand uncertainty.
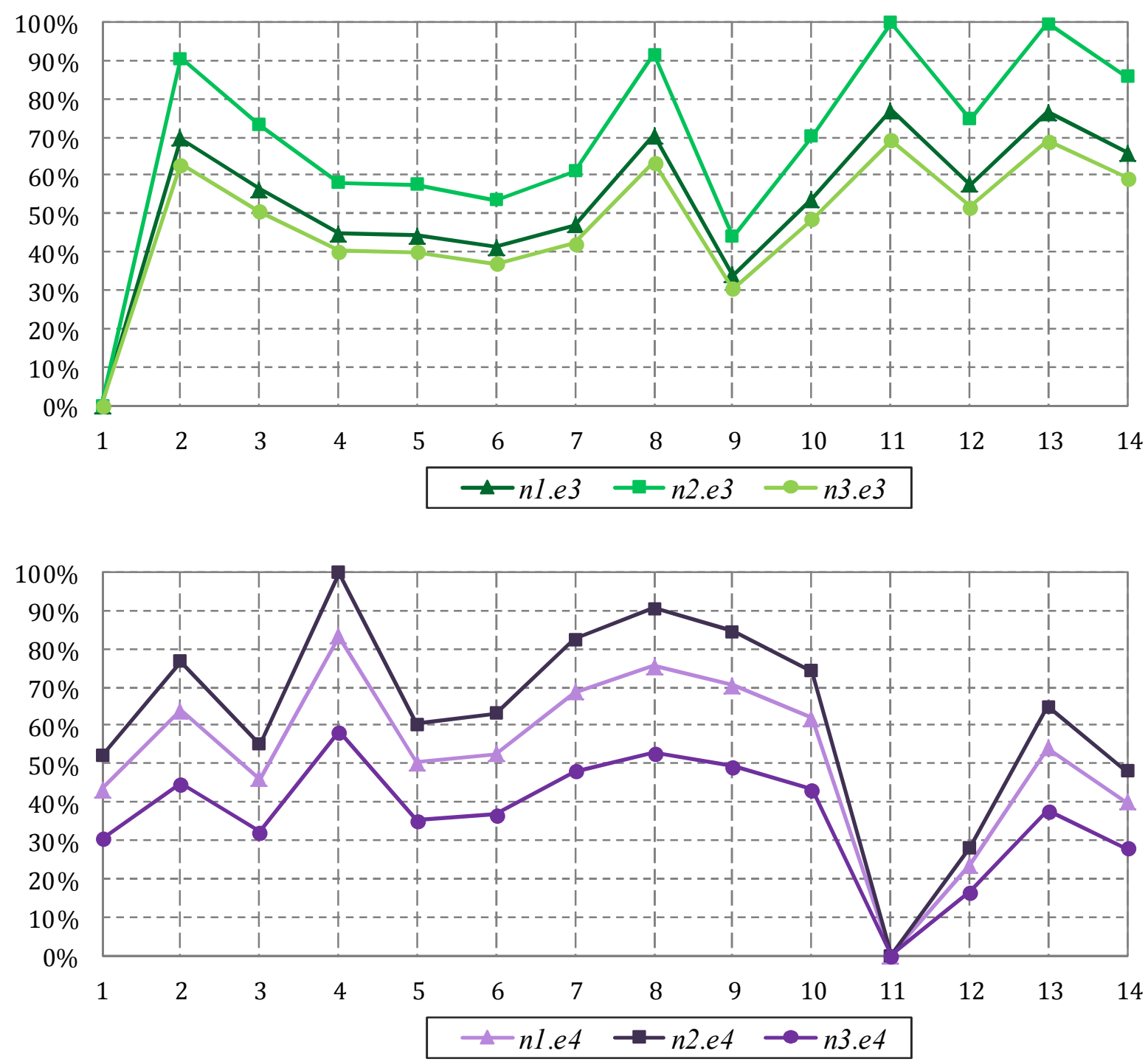

Figure 3: Case Study 1 - Normalized demand profiles for products per scenario.

\subsubsection{Case Study 1: Results.}

The resulting optimization model consists of 5,947 equations, 3,514 continuous variables and 923 binary variables. A zero optimality gap was reached after 43,202 CPU s. Figure 4 displays the optimal operational and cleaning plan for the production and utility systems. The utilization profile of cleaning resources is also shown there. Cleaning resources utilization has its peak in days 4 and 6 where three cleaning tasks take place in parallel. There are no offline cleaning tasks for the utility units $i 1, i 2$ and $i 5$, but a number of online cleaning tasks takes place in them. For instance, utility unit il undergoes its first online cleaning in day 6 and its second online cleaning in day 11, satisfying the minimum time between two consecutive online cleanings. A similar case is observed in utility unit $i 2$ where three online cleanings take place in days 4,9 and 14 . An online cleaning also is observed in day 2 for utility unit $i 5$. For utility unit $i 3$ and $i 4$ offline cleaning task option $q 2$ and $q 3$ start in day 3 and 9 , respectively. It is observed that utility unit $i 4$, which can only generate utility $e 1$, operates only from day 1 to day 5 . Although this utility unit does not operate again in the remaining planning horizon, an 
offline cleaning task takes place in latter periods so as to restore the efficiency of the unit and meet the terminal constraints related to its maximum degradation level at the end of the planning horizon. A similar case is observed for production unit $i 7$. Production units $i 8$ and i6 undergo offline cleaning tasks $q 1$ that start in day 1 and 4, respectively. As expected, all offline cleaning tasks for production units start within the predefined earliest and latest starting time (i.e., day 1 to 9). Finally, it is observed that production unit $i 6$ produces product $e 3$ and production unit is produces product $e 4$ in all their operating periods except for one time period.

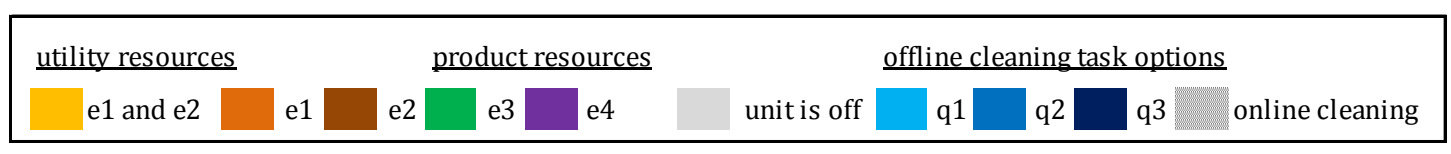
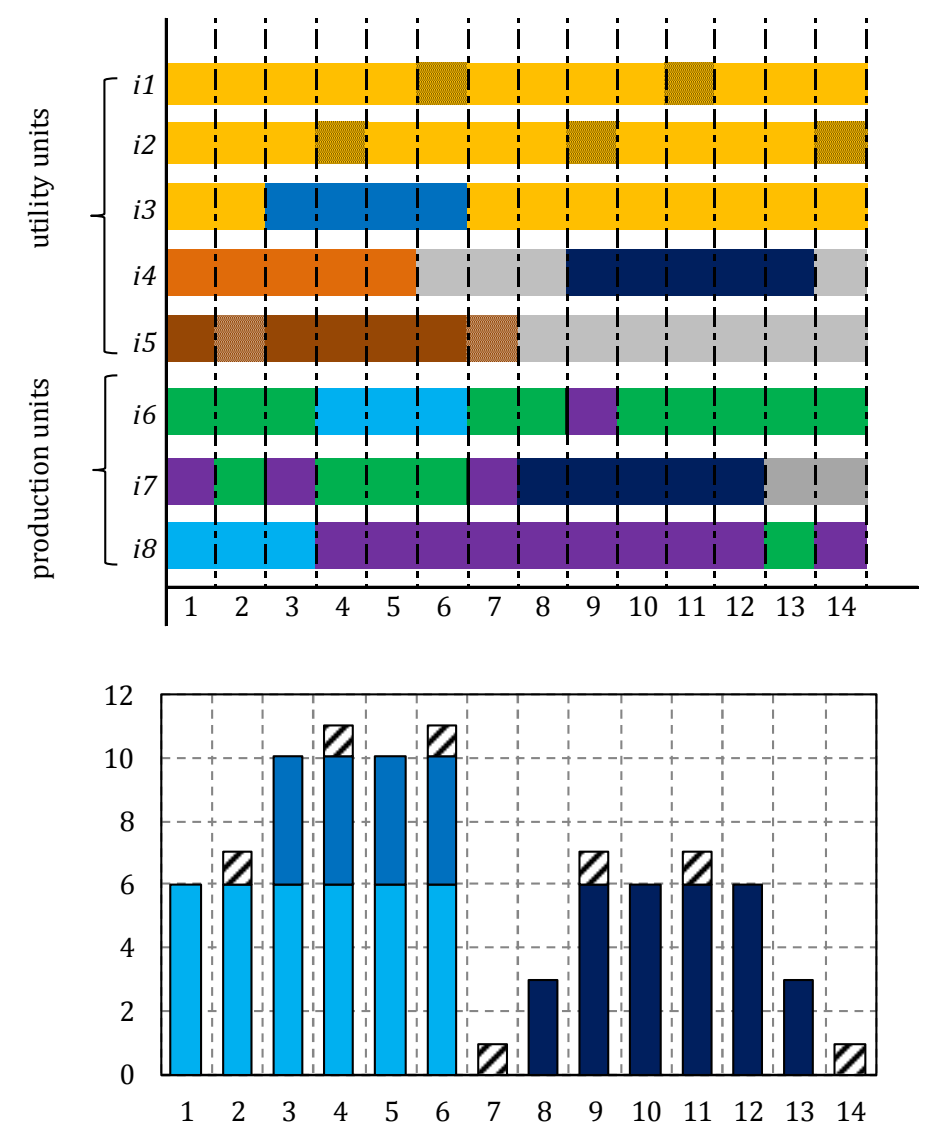

Figure 4: Case Study 1 - Optimal operational and cleaning plan for production and utility system and total cleaning resources utilization profile.

Figure 5 displays the normalized operating level profiles for utility and production units for each scenario, having as a reference the maximum operating level of each unit. In the utility system, utility unit $i 1$ operates at its maximum operating level for all scenarios throughout the planning horizon but in day 6 and 11 due to online cleaning (i.e., due to reduced operating capacity). In general for the scenarios considered, utility unit i2 operates near or at its maximum operating level for most of the planning horizon but in day 4,9 and 14 where online cleanings are observed. For all its operating time periods (i.e., excluding cleaning periods), utility unit $i 3$ operates at its maximum operating level in the high-demand scenario $n 2$, but it operates at its minimum operating level in the low-demand scenario $n 3$. This has been expected, since lower demand for products would result in lower requirements for utilities. 
Similar observations can be done for the remaining utility units. In the production system, production unit i6 operates in its maximum capacity in all its operating periods for all scenarios. Production unit is operates near or at its maximum capacity in most of its operating periods in scenarios $n 1$ and $n 2$, while many operating level fluctuations are observed in the low-demand scenario $n 3$. Production unit $i 7$ operates just half of the planning horizon and its operating level is near or at its minimum in most of its operating periods for scenario $n 1$, and near or at its maximum for the high-demand scenario $n 2$.

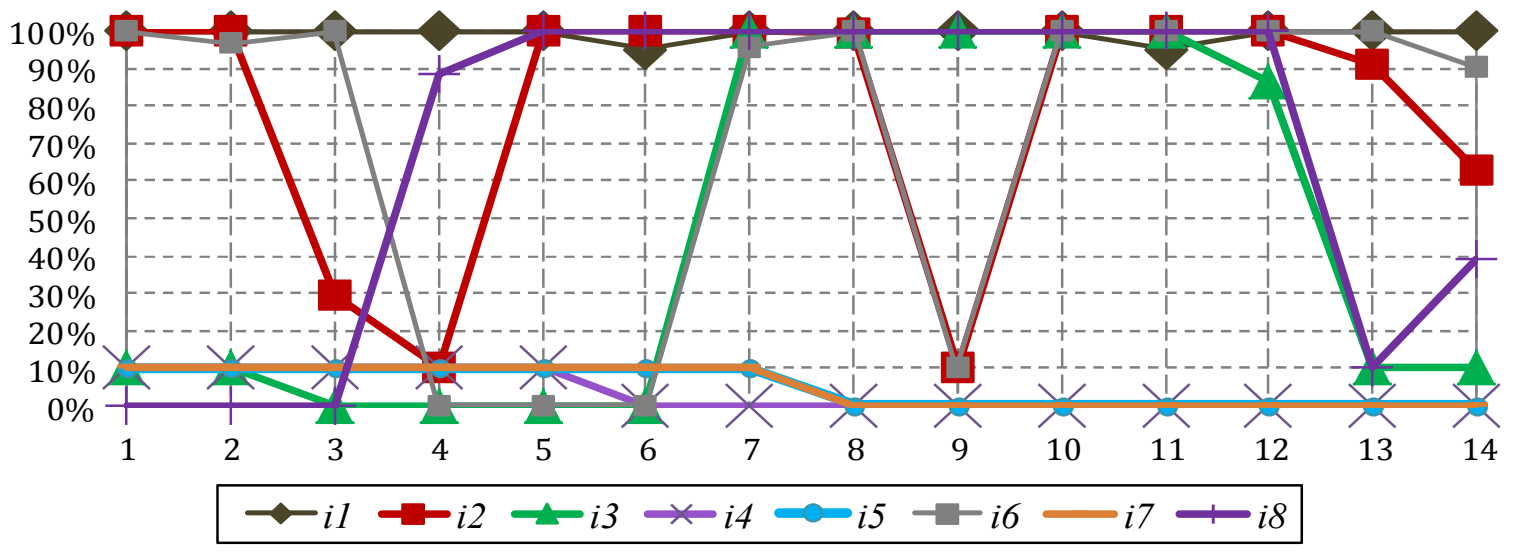

(a) Scenario $n 1$

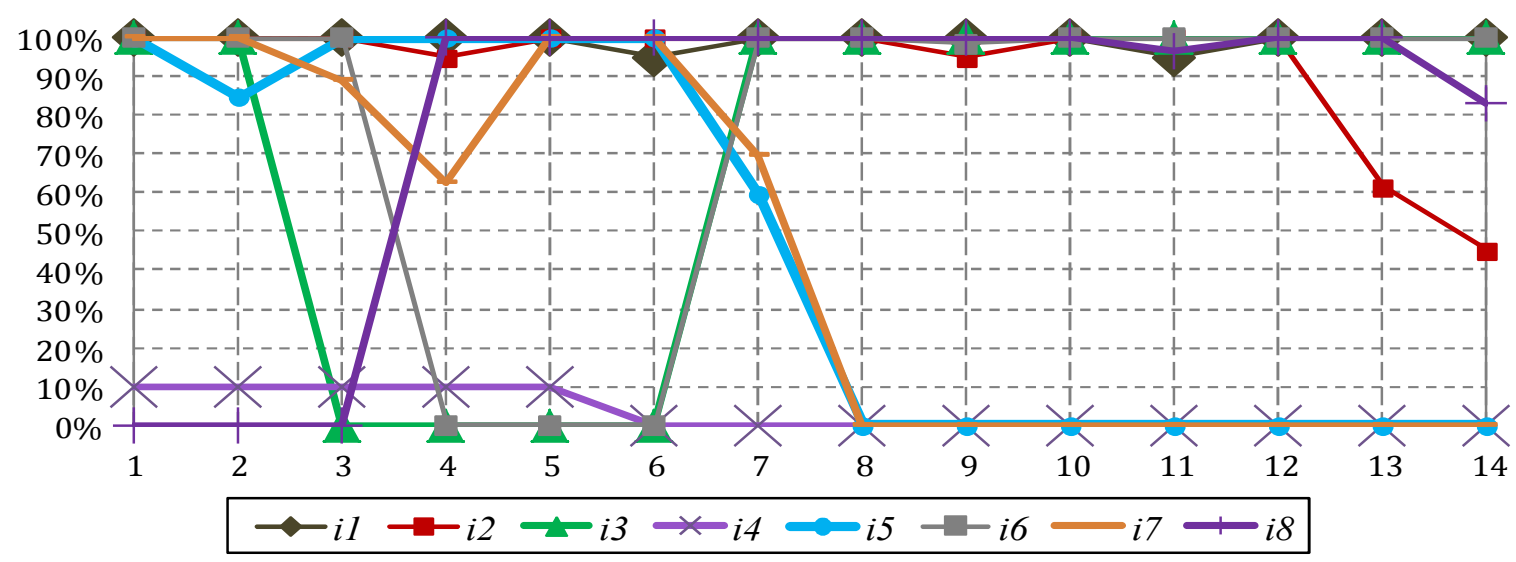

(b) Scenario $n 2$

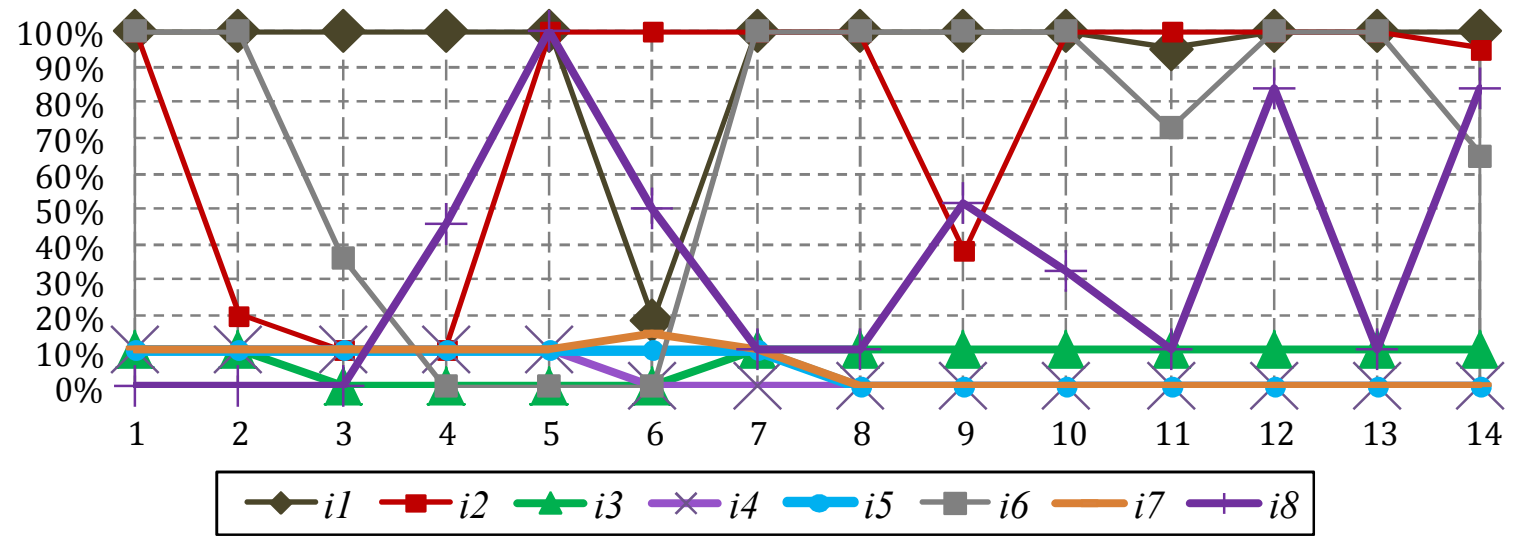

(c) Scenario $n 3$ 


\section{Figure 5: Case Study 1 - Normalized operating level profiles for utility and production units per scenario.}

Figure 6 displays the normalized total production profiles for each resource (utility or product) per scenario; calculating the aggregated production of each resource from each unit and divide it by the maximum production plant capacity for each resource. As expected, the production peak for resources is observed in the high-demand scenario $n 2$ followed by those in the medium-demand scenario $n l$ and low-demand scenario $n 3$. Generally speaking, the production level profiles for utilities $e 1$ and $e 2$ follow quite a similar trend at each scenario, mainly due to the three cogeneration utility units. Since production units could produce at most one product at a time, the total production profile for one product follows the opposite trend of that of the other product. In general, production peaks for one product result in production lows for the other. In all scenarios and for any product, its demand in zero or low total production periods is exclusively satisfied by the inventories, since no purchases of products have been reported.
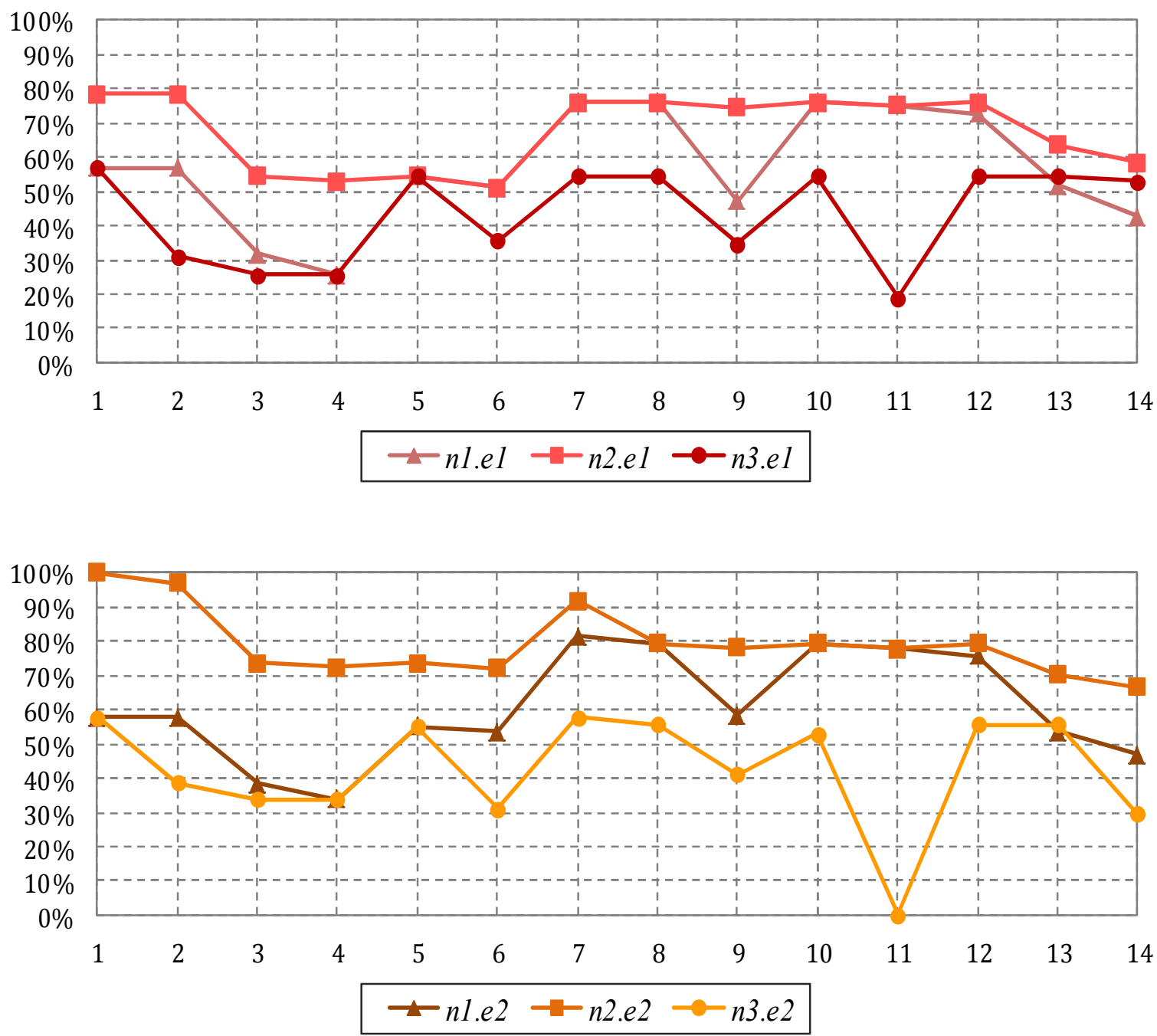

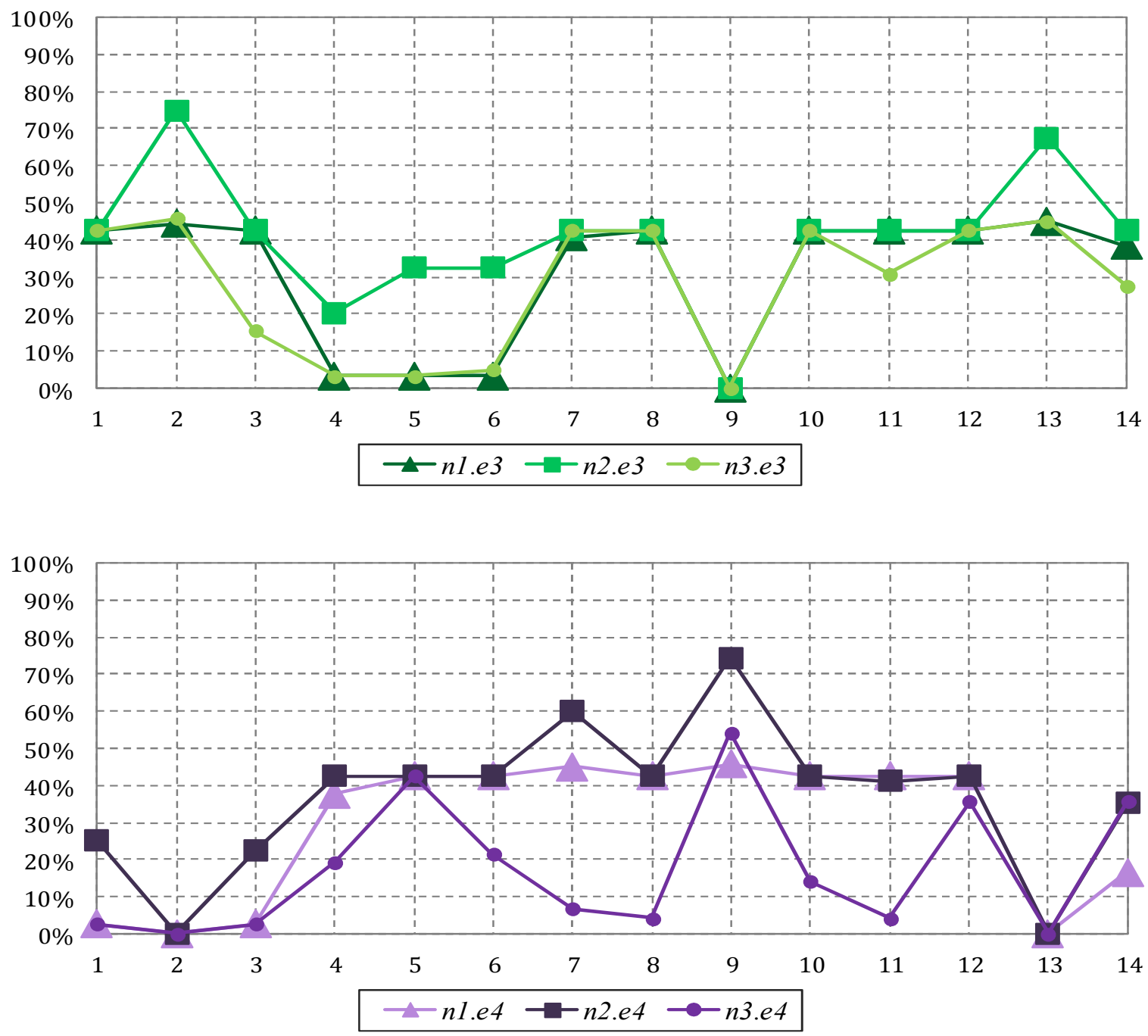

Figure 6: Case Study 1 - Normalized total production profiles for utilities and products per scenario.

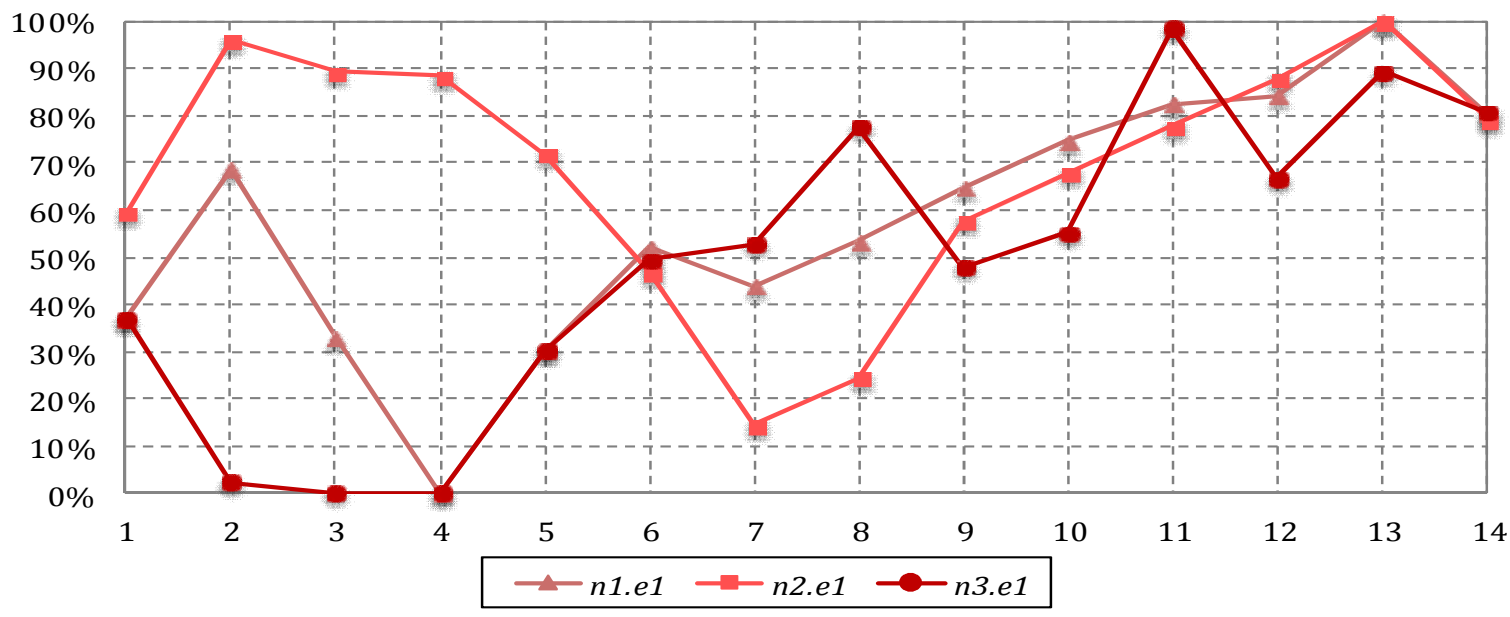



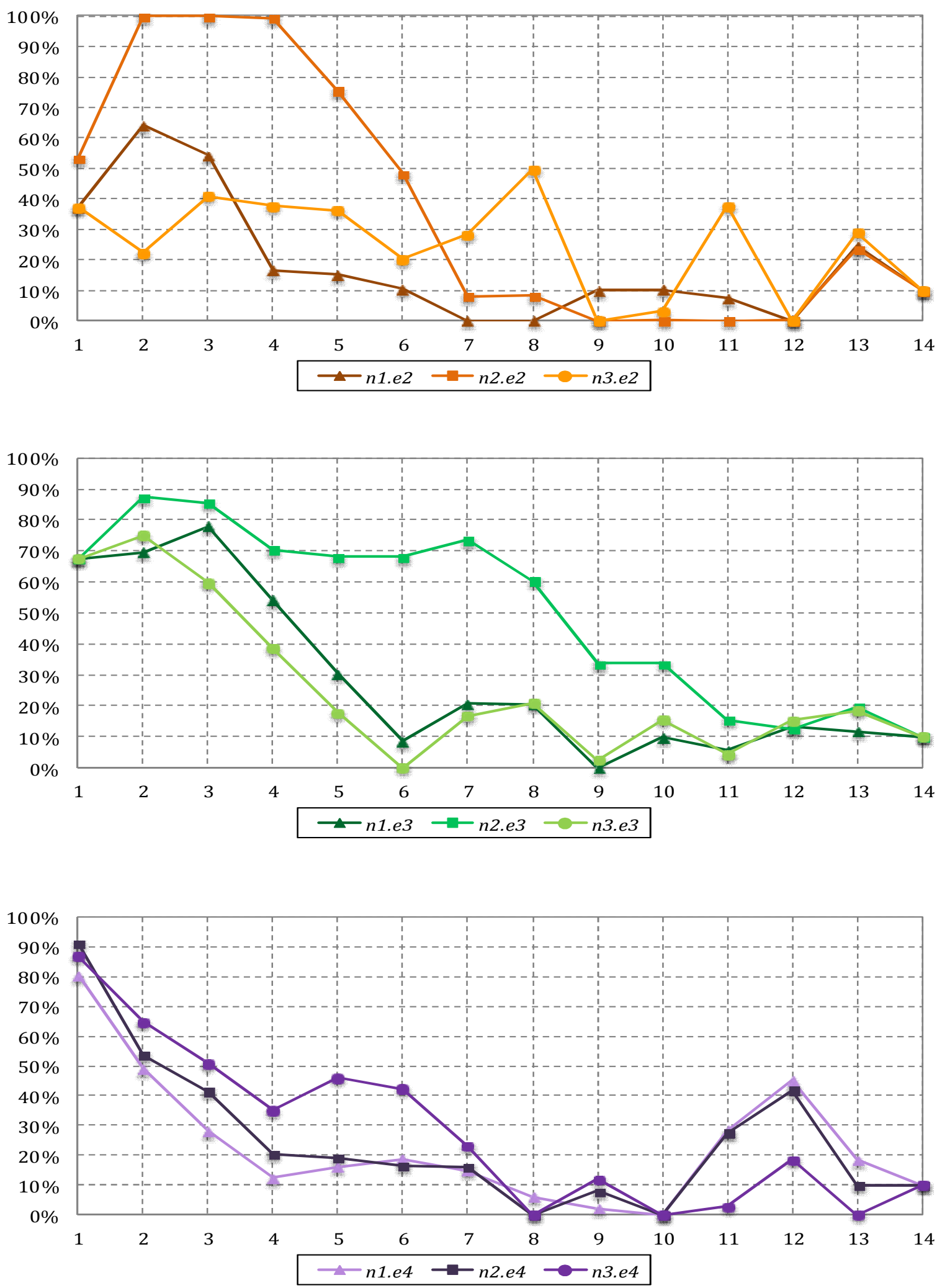

Figure 7: Case Study 1 - Normalized inventory profiles for utilities and products per scenario.

Figure 7 displays the normalized inventory profiles for utilities and products for each scenario, having as a reference the corresponding maximum inventory level of each inventory tank. For 
all scenarios at the end of the planning horizon, the inventory levels for utility $e 2$ and products $e 3$ and $e 4$ are $10 \%$ of their corresponding maximum inventory levels, which is equal to the lower bound of the imposed terminal constraints. However, the inventory level for utility el at the end of the planning horizon is around $80 \%$ of its maximum inventory level for all scenarios. This is an indirect result of the operation of the cogeneration units $i 1$ to $i 3$ that satisfy the much higher demand for utility $e 2$ in comparison with that for utility $e 1$, cogenerating excessive amount of utility $e l$ that is eventually stored.

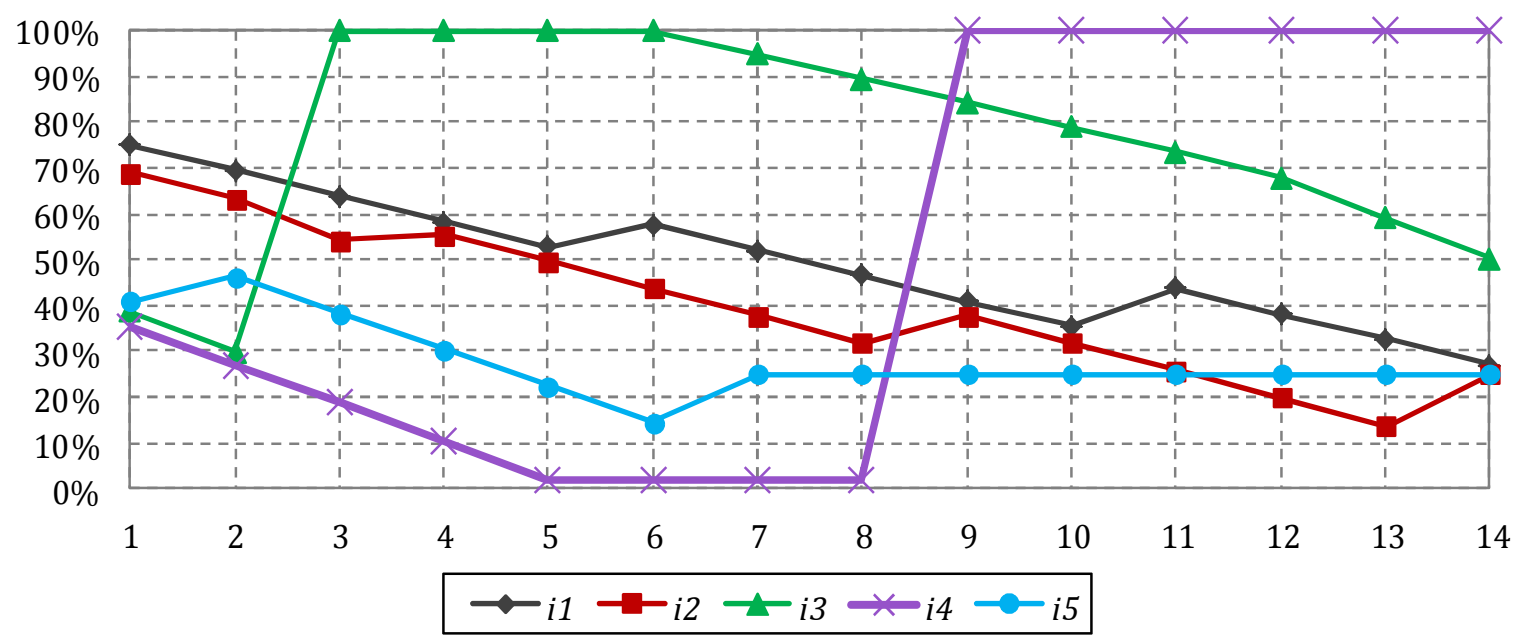

Figure 8: Case Study 1 - Performance level profiles for utility units for scenario $n 1$.

Figure 8 shows the performance level profiles for utility units that are subject to conditionbased cleaning for medium-demand scenario $n 1$. Recall that the performance level of a unit depends on its cumulative time of operation (first-stage variables) and its cumulative operating level deviation (second-stage variables). The performance level profile for other scenarios follows a quite similar trend because the cumulative time of operation is the same for all scenarios (i.e., scenario-independent) and only the cumulative operating level deviation may be different among the scenarios. However, the performance level profiles are almost the same for all scenarios since utility units tend to operate at their maximum load in most their operating periods (see Figure 5). Utility units $i 3$ and i4 fully recover their performance though offline cleaning. Also, it can be observed the partial performance recovery of utility units $i 1$, $i 2$ and $i 5$ through online cleanings as shown in: (i) day 6 and 11 for utility unit $i 1$, (ii) day 4,9 and 14 for utility unit $i 2$, and (iii) day 2 and 7 for utility unit $i 5$. At the end of day 14 , the performance levels of all operating utility units (i1, i2 and $i 3)$ and non-operating utility units ( $i 4$ and $i 5$ ) remain above $25 \%$, satisfying the terminal constraints imposed. 


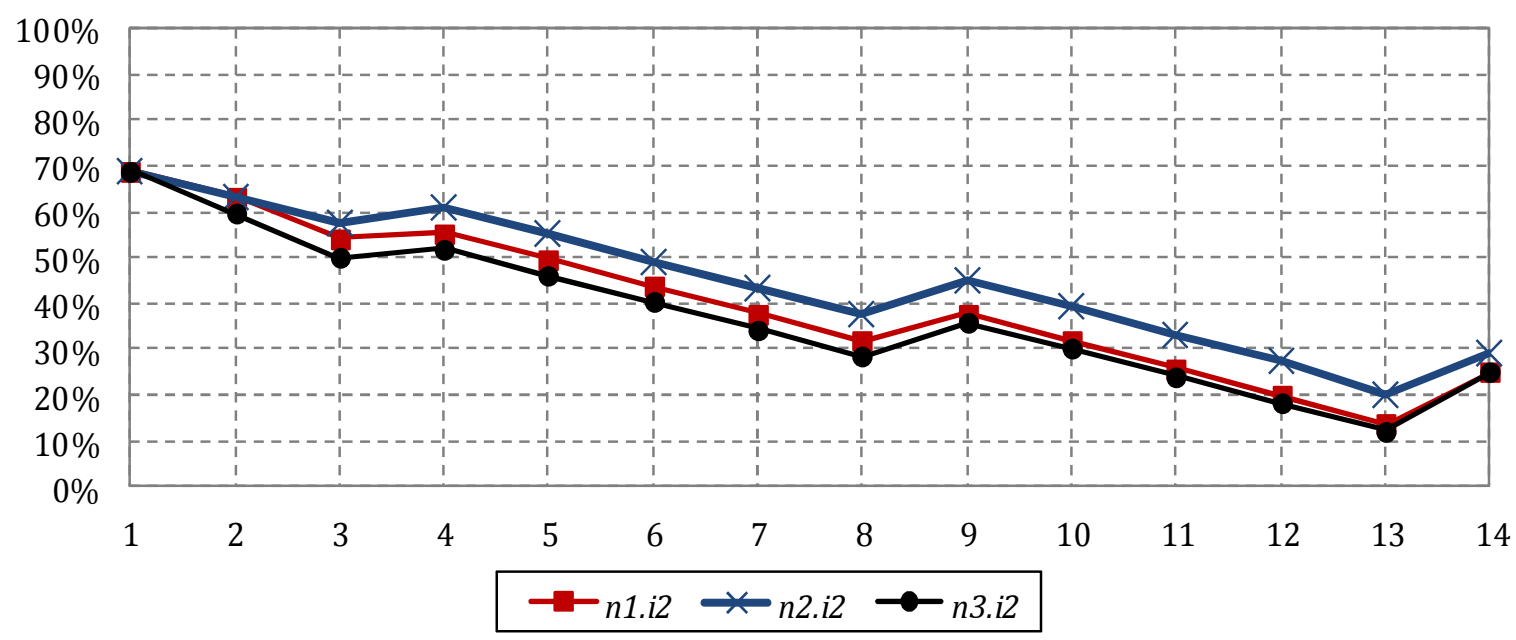

Figure 9: Case Study 1 - Performance level profile for utility unit $i 2$ per scenario.

Figure 9 shows the performance level profile for utility unit i2 per scenario. The highest performance level profile for this unit is observed for the high-demand scenario $n 2$ which is due to its reduced cumulative operating level deviation since it operates at closer or at its maximum load in most of its operating periods in comparison with the other two scenarios (see Figure 6). Recall that the reference operating load for any unit is equal to its maximum operating level.

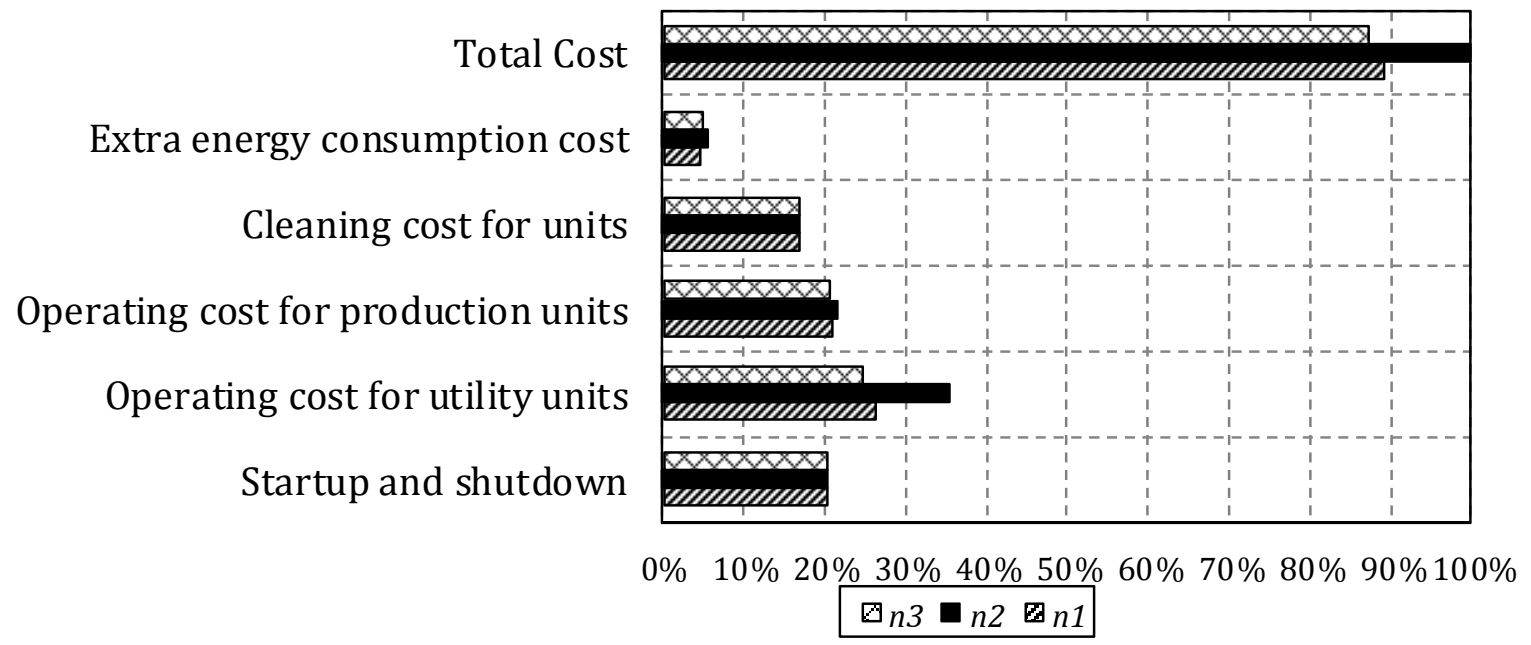

Figure 10: Case Study 1 - Cost breakdown comparison per scenario.

Figure 10 shows the cost breakdown comparison among all scenarios. Each cost term for each scenario is divided by the total cost of high-demand scenario $n 2$ which reports the highest total cost than the other scenarios considered. The costs terms consist of: (i) fixed and varied operating cost for utility units, (ii) fixed and varied operating cost for production units, (iii) extra power consumption cost, (iv) cleaning cost for units, and (v) startup and shutdown cost. The major cost difference is observed in the operating cost for utility units in scenario $n 2$ which is $25.4 \%$ and $30.2 \%$ higher than that in scenario $n 1$ and scenario $n 3$, respectively. In addition, the operating cost for production units in scenario $n 2$ is $3.7 \%$ and $5.3 \%$ higher than that in scenario $n 1$ and $n 3$, respectively. Extra energy consumption in scenario $n 2$ is $20.0 \%$ and $14.1 \%$ than that in scenario $n 1$ and $n 3$, respectively. Startup/shutdown and cleaning 
costs are the same for all scenarios, since they involve only scenario-independent first-stage decision variables. Total cost in high-demand scenario $n 2$ is $10.9 \%$ and $12.6 \%$ higher than that in medium-demand scenario $n 1$ and low-demand scenario $n 3$, respectively.

\subsection{Case Study 2: Integrated Planning of Production and Utility Systems via a Rolling Horizon Stochastic Programming Approach}

This case study presents an application of the rolling horizon stochastic programming approach proposed in this study for a slight variation of the integrated condition-based planning of production and utility systems addressed in the previous case study. A two-stage scenario-based stochastic programming method is followed.

\subsubsection{Case Study 2: Description.}

The plant layout as well as main parameters and operational costs are the same as in the previous case study. Terminal constraints, cleaning resources availability and alternative cleaning options are also the same as before. The initial state of the overall system at the beginning of planning horizon is the similar to that of Case Study 1 (see Table 1). In contrast to the previous case study, here all production and utility units are subject to condition-based cleaning policies. Also here the minimum time between two consecutive online cleanings in a unit $\left(\gamma_{i}^{o n}\right)$ is five and six time periods for utility and production units, respectively. A total planning horizon of 28 day time periods is considered here. The demand profiles for products are displayed in Figure 11.

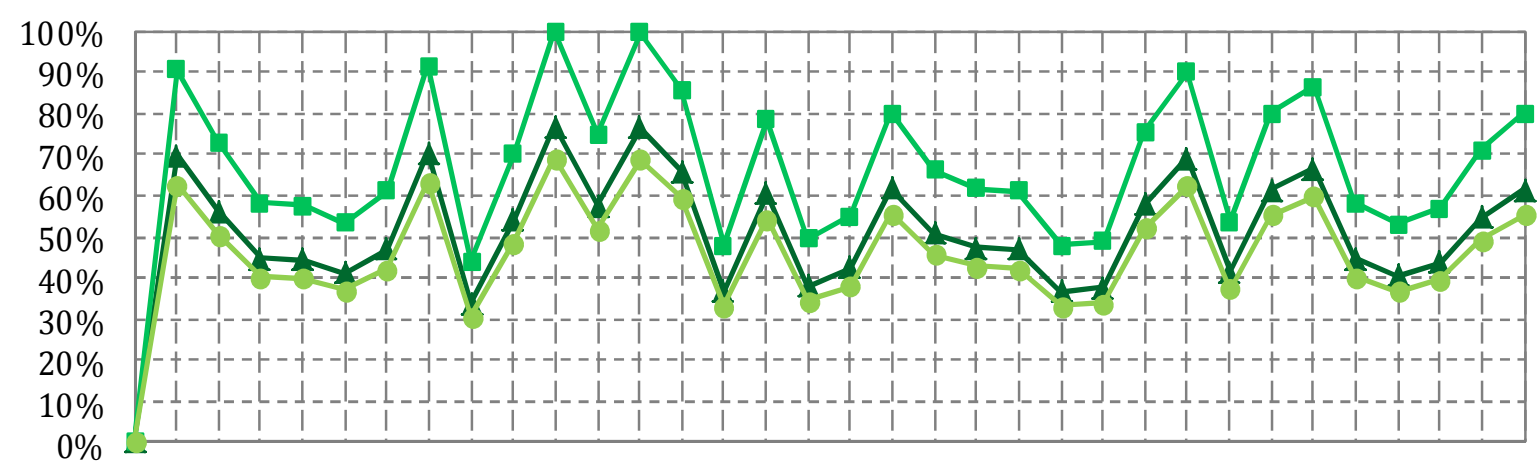

$122 \quad 3 \quad 4 \quad 5 \quad 6 \quad 7 \quad 8 \quad 910111213141516171819202122232425262728293031323334$ $\rightarrow-n 1 . e 3-n 2 . e 3-n 3 . e 3$

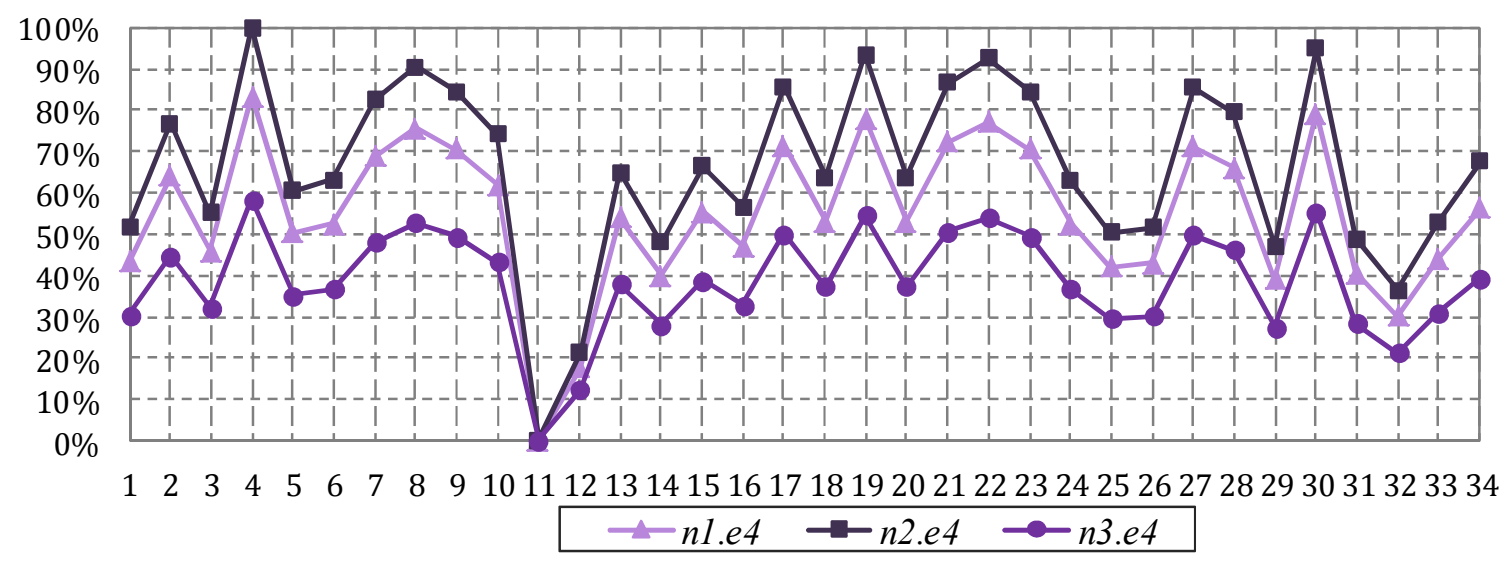

Figure 11: Case Study 2 - Rolling Horizon Stochastic Integrated Approach: Normalized demand profiles for products per scenario. 
For the rolling horizon approach, a prediction horizon equal to seven time periods and a single-period control horizon has been used. A total number of 28 iterations has been solved. For each iteration, the integrated planning problem for the next seven time periods is solved through the two-stage scenario-based stochastic programming model. After each iteration, a planning problem for a new prediction horizon is solved by moving forward the planning horizon by the length of the control horizon considered. Although we obtain solutions for all scenarios considered, in reality only one can occur after each iteration (under the assumption that exactly one scenario of the ones considered must occur), and we refer to this as active scenario. Only the solution of the control horizon of the active scenario of the current prediction horizon is applied after each iteration, and therefore the initial state of the overall system for the next prediction horizon is updated according to the solution of the active scenario in the previous iteration. Note that active scenario is the realized demand scenario of the control horizon of interest that takes into account the solution of first-stage decision variables for all scenarios considered in the previous iteration. In this case study, parameters that need to be updated according to the solution of active scenario are: (i) the level of every inventory tanks; and (ii) the deviation of the operating level per unit. Other parameters that do not depend on active scenario are the solution of the first-stage decision variables such as: (i) the current operating status of each unit; (ii) the startup and shutdown history of units; (iii) the cumulative time of operation per unit; and (iv) the offline and online cleaning history of units. We assume that the active scenario of an iteration is not known just before solving the planning problem of the next iteration. Table 2 presents the active scenario for each iteration

Table 2. Case Study 2: Active scenario per iteration.

\begin{tabular}{|c|c|c|c|c|c|c|c|}
\hline \multirow[b]{2}{*}{ Iteration } & \multicolumn{3}{|c|}{ Active Scenario } & \multirow[b]{2}{*}{ Iteration } & \multicolumn{3}{|c|}{ Active Scenario } \\
\hline & $n 1$ & $n 2$ & $n 3$ & & $n 1$ & $n 2$ & $n 3$ \\
\hline 1 & & & $\mathrm{x}$ & 15 & & $\mathrm{x}$ & \\
\hline 2 & $x$ & & & 16 & & & $x$ \\
\hline 3 & & $x$ & & 17 & & $x$ & \\
\hline 4 & & & $\mathrm{x}$ & 18 & & $x$ & \\
\hline 5 & & & $\mathrm{x}$ & 19 & $\mathrm{x}$ & & \\
\hline 6 & & $x$ & & 20 & & $x$ & \\
\hline 7 & & & $\mathrm{x}$ & 21 & & $x$ & \\
\hline 8 & $x$ & & & 22 & & & $x$ \\
\hline 9 & $x$ & & & 23 & $\mathrm{x}$ & & \\
\hline 10 & $x$ & & & 24 & $x$ & & \\
\hline 11 & & $x$ & & 25 & $x$ & & \\
\hline 12 & $x$ & & & 26 & $\mathrm{x}$ & & \\
\hline 13 & & & $\mathrm{x}$ & 27 & $\mathrm{x}$ & & \\
\hline 14 & $x$ & & & 28 & $\mathrm{x}$ & & \\
\hline
\end{tabular}

\subsubsection{Case Study 2: Results.}

On average, each optimization model consists of 4,020 equations, 2,101 continuous variables and 532 binary variables. The average computational time is 3,274 CPU s. Figure 12 displays how the final operational and cleaning plan for the 28-day horizon is constructed through the rolling horizon approach. An illustrative example of the first three iterations is presented. The last Gantt chart shows the implemented operational and cleaning plan and the total utilization profile of cleaning resources for the planning horizon considered. Notice that the implemented Gantt chart is applicable for all scenarios considered, since all binary decisions variables related to the operational and cleaning status of the units are considered as first-stage variables in the stochastic programming model. For the first iteration, a planning problem is 
solved for time periods 1 to 7 and the solution of the active scenario of the first time period is saved. For the second iteration, a new planning problem for time periods 2 to 8 is solved by updating the initial state according to the active scenario of the first iteration. This receding horizon scheme continues until all 28 iterations are solved.

According to Figure 12, 4 offline and 14 online cleaning tasks for utility and production units are reported. The maximum total utilization of cleaning resources is observed in time period 14 where: (i) 8 cleaning resources are needed for two offline cleaning options $q 2$ in unit $i 2$, (ii) 3 cleaning resources for offline cleaning option $q 3$ at unit $i 3$ and, (iii) one cleaning resource for the online cleaning of unit $i 1$. Simultaneous online cleanings are observed for utility unit $i 5$ and production unit $i 6$ in the fourth time period. Utility unit $i 4$, which can only produce utility el operates just from day 1 to 5 because cogeneration utility units $i 1, i 2$ and $i 3$ could not fully satisfy the demand for utility $e 1$ at this time horizon. Utility unit $i 5$, which can produce only utility $e 2$ operates from day 25 to 28 to satisfy the needs for utility $e 2$ because utility unit $i 1$ is closed on these days. In general, production unit $i 7$ has the highest operational costs in comparison with the other production units. Since the other two production units can satisfy the demand for products for the planning horizon considered, production unit $i 7$ remains idle throughout the planning horizon but not in day 1 , where it operates due to the minimum run constraint (see Figure 12). 


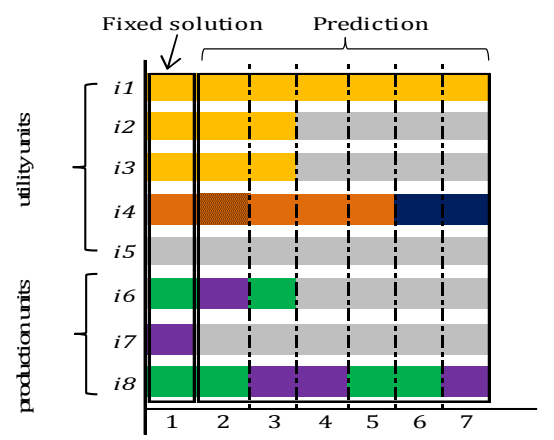

Iteration 1

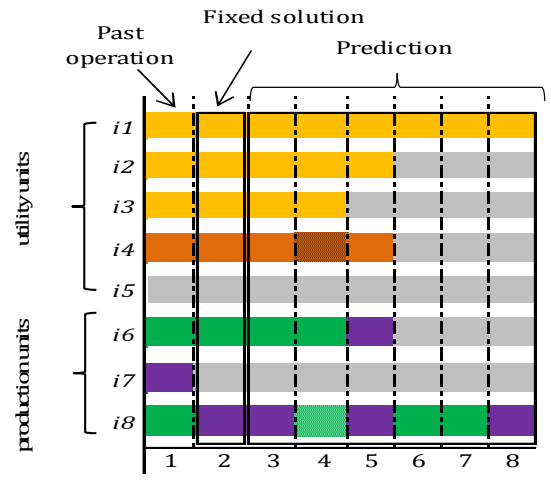

Iteration 2

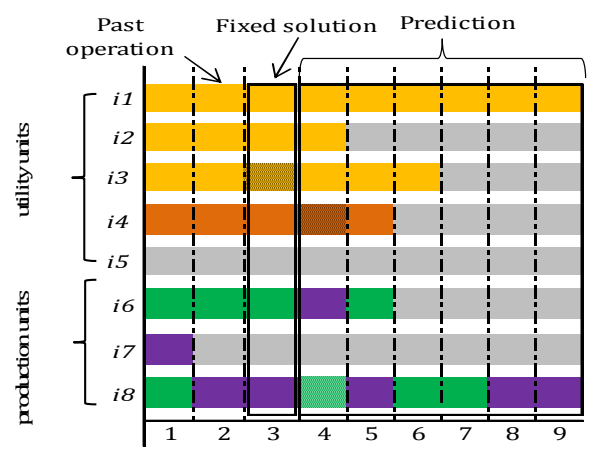

Iteration 3
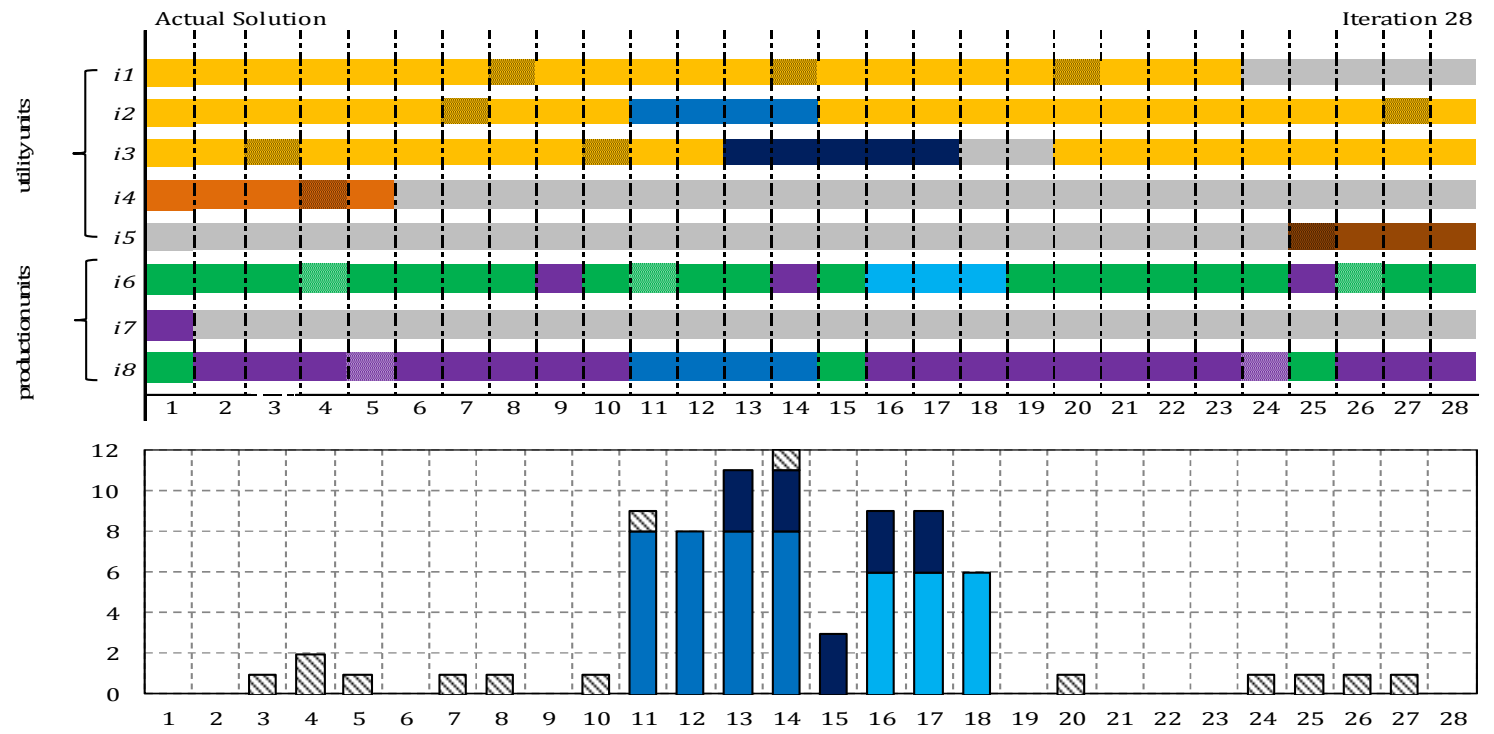

Figure 12: Case Study 2 - Rolling Horizon Stochastic Programming Approach: Plan generation via rolling horizon and total utilization profile of cleaning resources. 


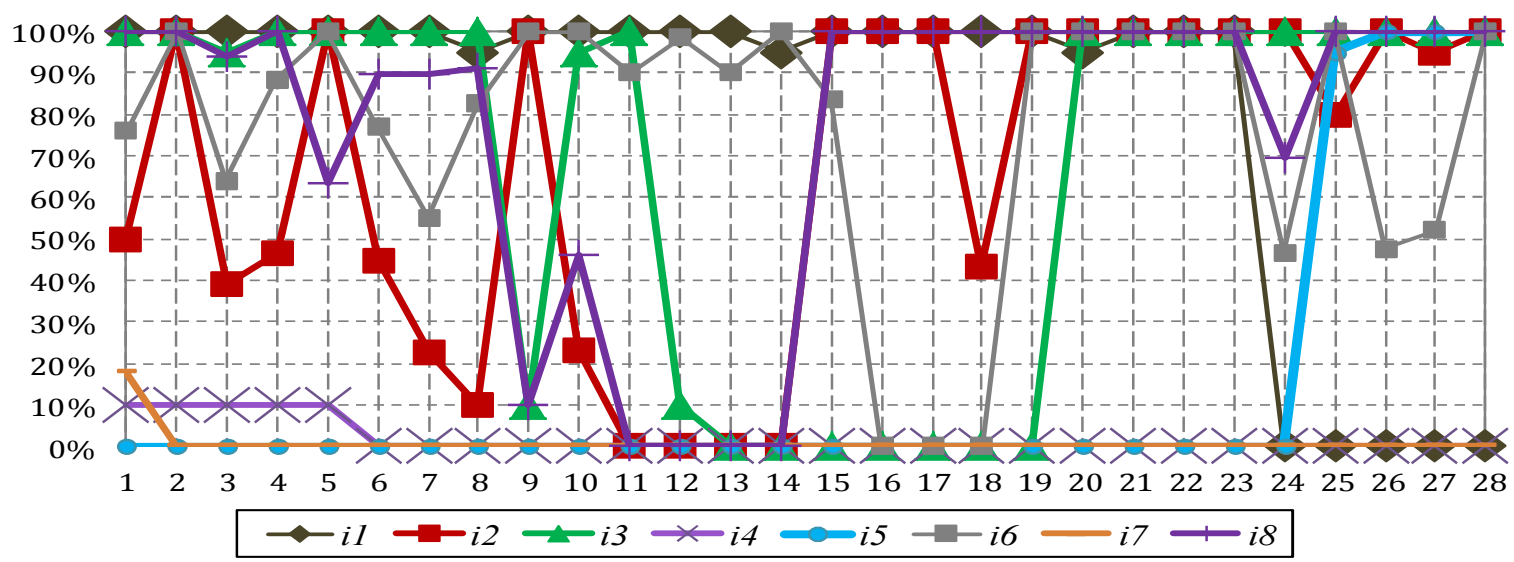

(a) Scenario $n 1$

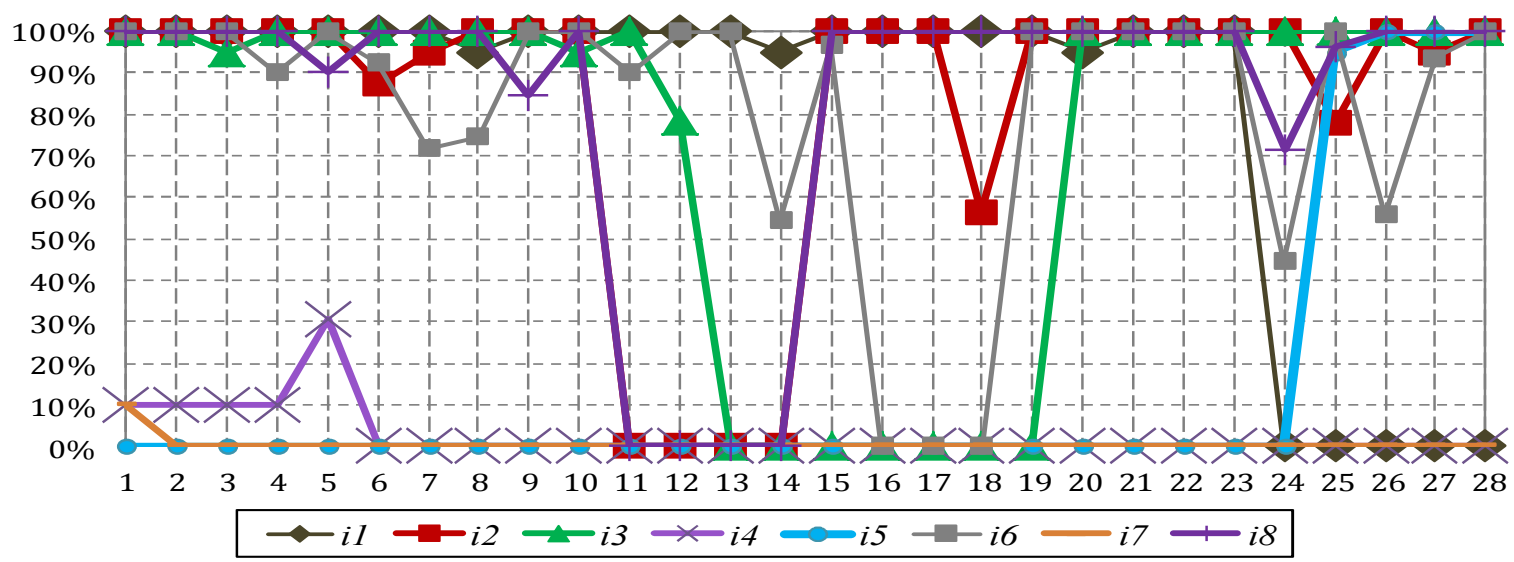

(b) Scenario $n 2$

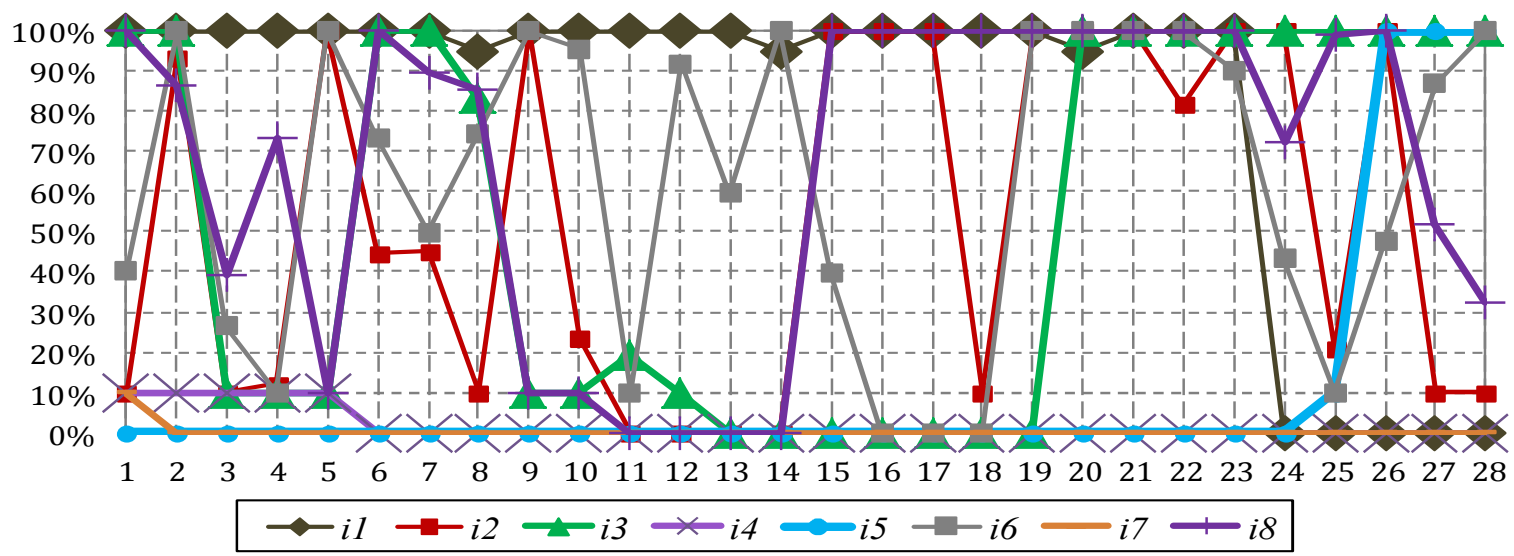

(c) Scenario $n 3$

Figure 13: Case Study 2 - Rolling Horizon Stochastic Programming Approach: Normalized operating level profiles for utility and production units per scenario.

Figure 13 shows the normalized operating level profiles per scenario for all units. In the utility system, cogeneration utility unit $i 1$ operates very close or at its maximum operating level until day 23. Cogeneration utility units $i 2$ and $i 3$ operate at varied operating levels satisfying the fluctuations of the utilities requirements. Utility unit $i 4$, which can generate only utility $e 1$, operates (for just five time periods) at its minimum operating level in all scenarios, while utility 
unit $i 5$, which can only generate utility $e 2$, operates at its maximum operating level at its limited operating period (from day 25 to 28 ).
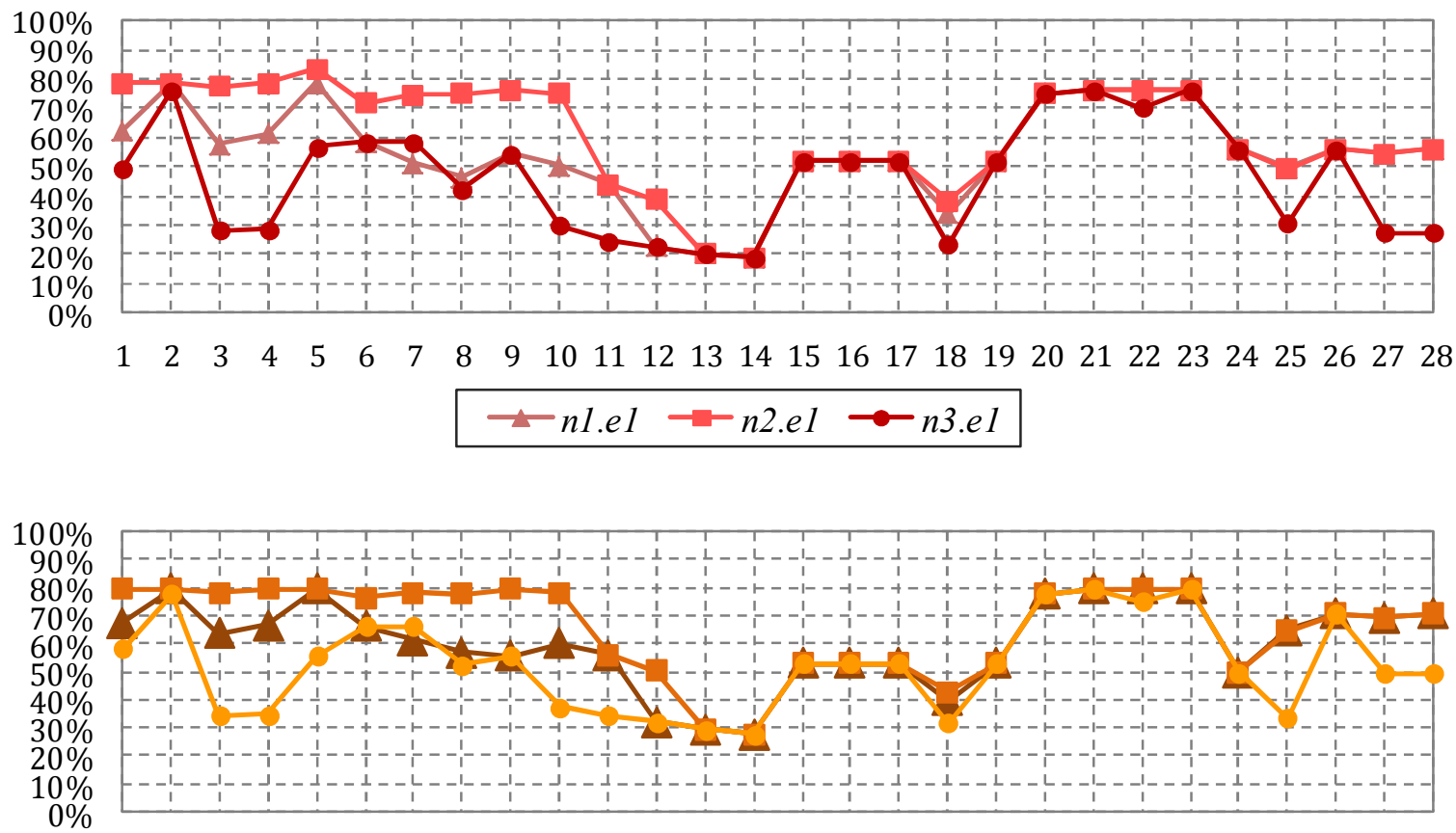

$\begin{array}{llllllllllllllllllllllllllll}1 & 2 & 3 & 4 & 5 & 6 & 7 & 8 & 9 & 10 & 11 & 12 & 13 & 14 & 15 & 16 & 17 & 18 & 19 & 20 & 21 & 22 & 23 & 24 & 25 & 26 & 27 & 28\end{array}$ $\rightarrow n 1 . e 2-n 2 . e 2 \rightarrow n 3 . e 2$

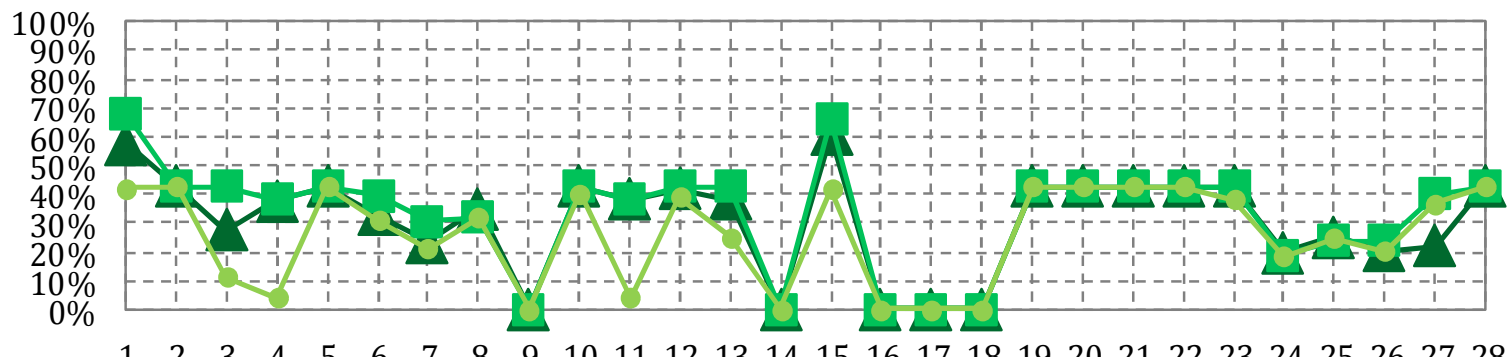

$\begin{array}{llllllllllllllllllllllllllll}1 & 2 & 3 & 4 & 5 & 6 & 7 & 8 & 9 & 10 & 11 & 12 & 13 & 14 & 15 & 16 & 17 & 18 & 19 & 20 & 21 & 22 & 23 & 24 & 25 & 26 & 27 & 28\end{array}$

$\multimap-n 1 . e 3 \multimap-n 2 . e 3 \multimap n 3 . e 3$

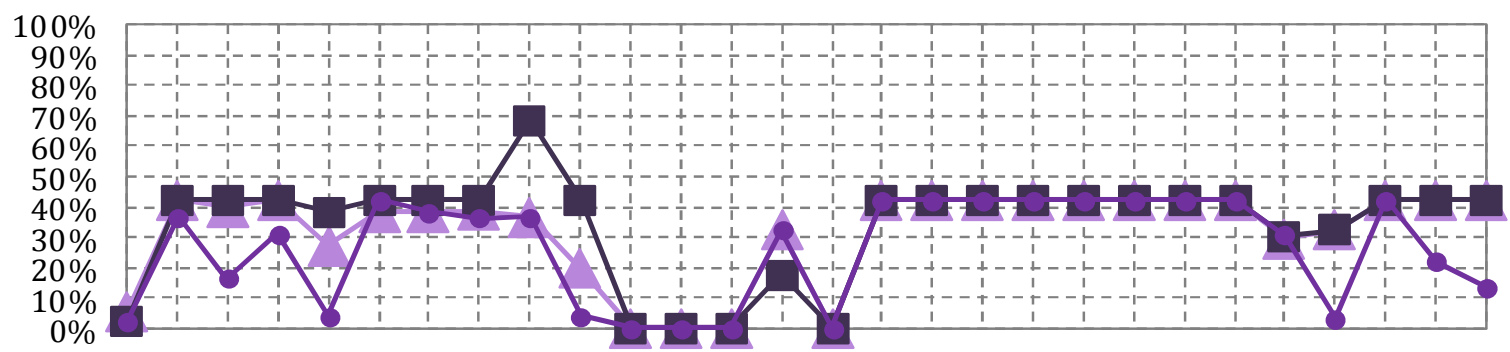

$\begin{array}{llllllllllllllllllllllllllll}1 & 2 & 3 & 4 & 5 & 6 & 7 & 8 & 9 & 10 & 11 & 12 & 13 & 14 & 15 & 16 & 17 & 18 & 19 & 20 & 21 & 22 & 23 & 24 & 25 & 26 & 27 & 28\end{array}$

$\rightarrow-n 1 . e 4 \rightarrow-n 2 . e 4 \rightarrow n 3 . e 4$

Figure 14: Case Study 2 - Rolling Horizon Stochastic Programming Approach: Normalized total production profiles for utilities and products per scenario. 
Figure 14 displays the normalized total production profiles for each utility and product for all scenarios. Similar observations can be made as in the previous case study. Production level trends are observed for utility resources $e 1$ and $e 2$ for all considered scenarios because there are three cogeneration utility units (i.e., $i 1, i 2$ and $i 3$ ). In general, the highest production profiles for both utilities throughout the planning horizon is observed in high-demand scenario $n 2$. The production peak for product $e 3$ is observed in day 15 for all considered scenarios, because two production units (i.e., i6 and $i 8$ ) operating at their high operating levels produce this product at in this time period. A similar observation can be made for product resource $e 4$ in day 9.
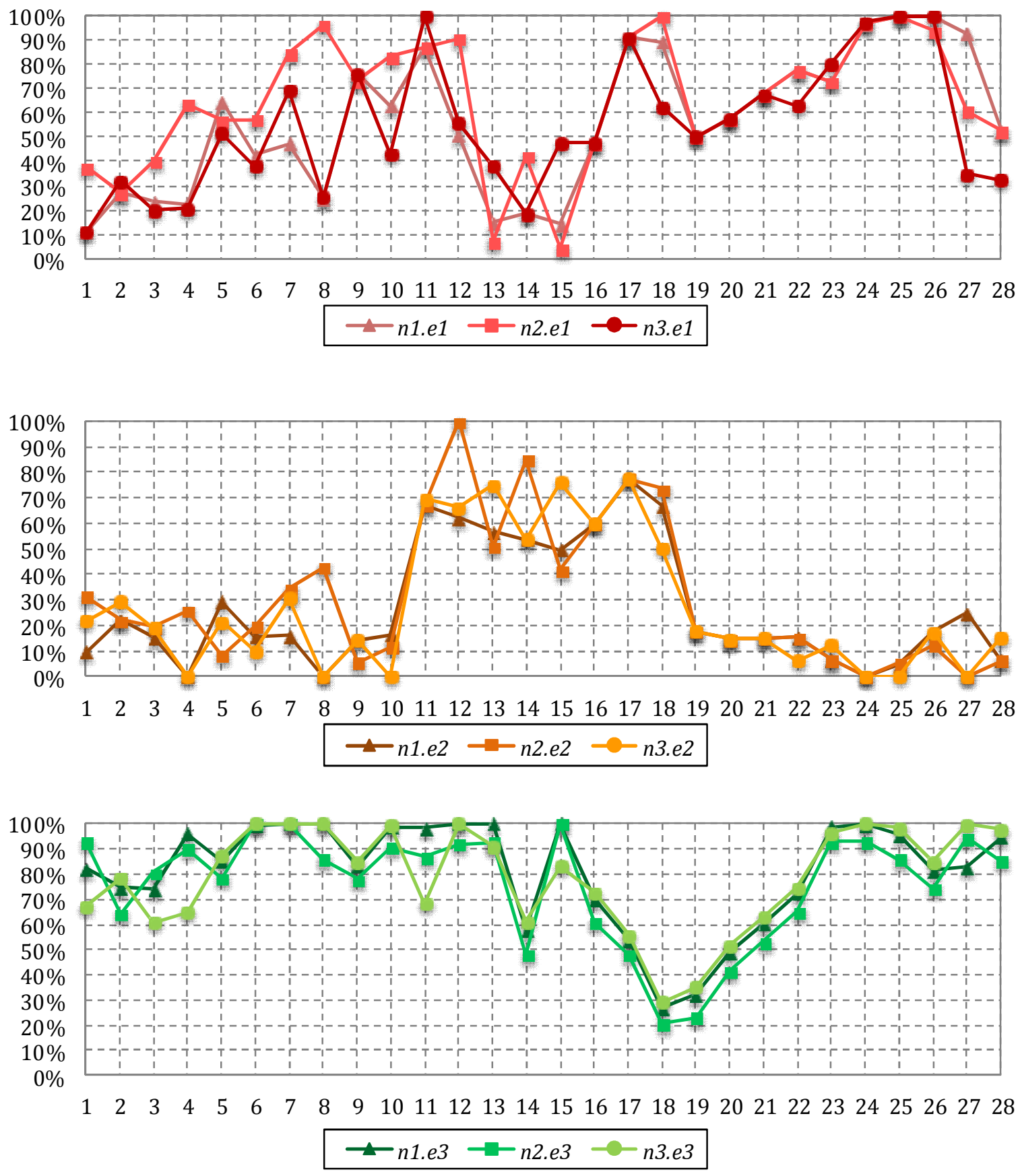


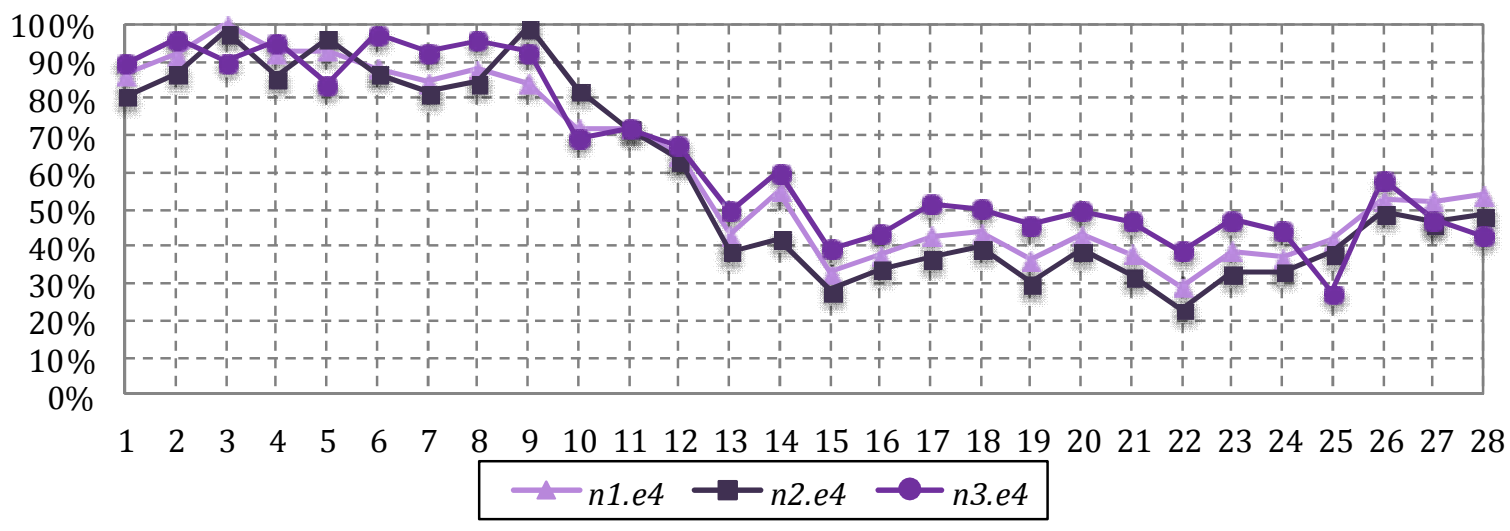

Figure 15: Case Study 2 - Rolling Horizon Stochastic Integrated Approach: Normalized inventory profiles for utilities and products per scenario.

Figure 15 displays the normalized inventory profiles for utilities and products, having as reference the corresponding maximum inventory level of each inventory tank. Low inventory levels for utility $e 1$ is observed for all scenarios from day 13 to 15 , because of the simultaneous multiple cleaning tasks in the cogeneration units at those periods (see Figure 12). High inventory levels for utility $e 2$ is reported for all scenarios from day 11 to 18 due to low utility demand at these time periods, because of the offline cleanings taking place in some production units (see Figure 12). For all scenarios, low inventory levels for product e3 are observed from day 16 to 18 because no production of product e3 takes place then. The inventory level for product $e 4$ reduces from day 11 to 15 due to the very limited production of product $e 4$ occurs in this time period (see Figure 12 and Figure 14). In general, inventory levels for both products in the low-demand scenario $n 3$ are slightly higher than those of other scenarios. It is important to recall that all inventory levels are subject to terminal constraints (i.e., higher than $10 \%$ of the maximum capacity of its inventory tank). For some scenarios, the inventory level for utility $e 2$ in day 28 is below $10 \%$. It should be clear that this is not a violation of the terminal constraint. The solution of day 28 (i.e., iteration 28) is derived by solving the planning problem for a prediction horizon from day 28 to day 34 , and for that planning problem the terminal constraint is satisfied in the last time period of the prediction horizon considered (i.e, day 34 and not day 28 ).

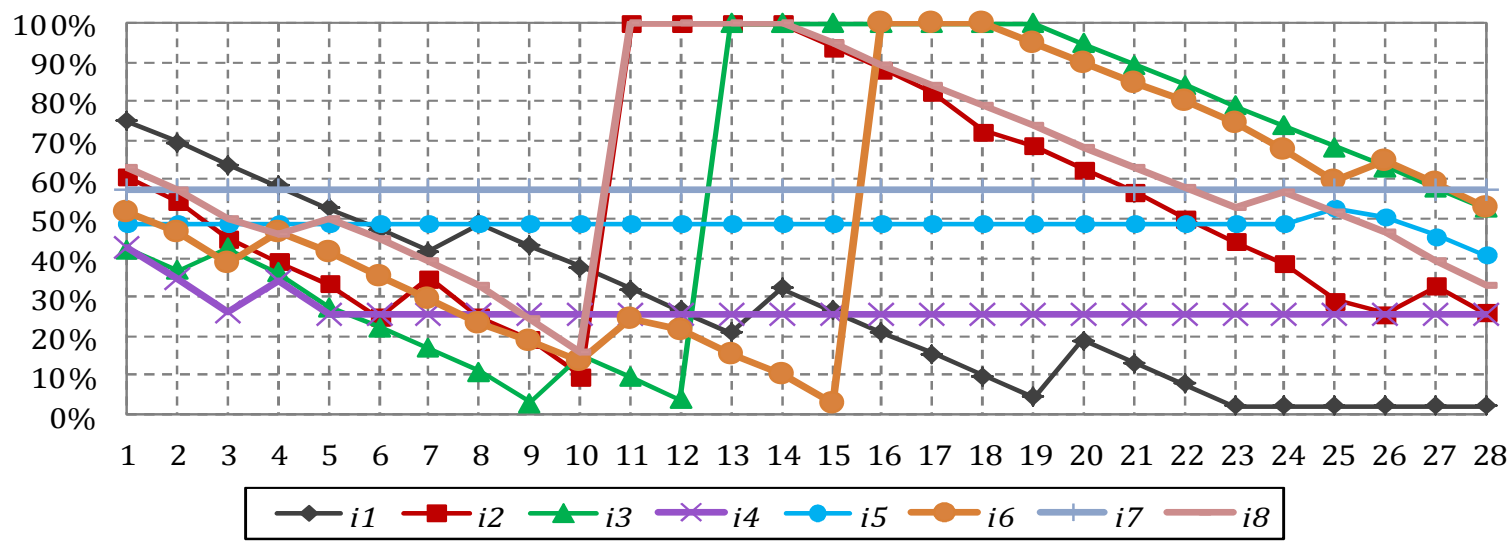

Figure 16: Case Study 2 - Rolling Horizon Stochastic Programming Approach: Performance level profiles for utility and production units for scenario $n 3$. 
The performance level profiles for utility and production units for scenario $n 3$ are displayed in Figure 16. Recall that the performance level of a unit depends on its cumulative time of operation and its cumulative operating levels deviation. Similar to Case Study 1, performance level profiles for the other scenarios are about the same. The performance of some utility units (i.e., i2 and i3) and production units (i.e., i6 and i8) is fully recovered once an offline cleaning occurs. It is also shown how a unit partially recovers its performance through online cleaning. For instance, unit $i 1$ partially recovers its performance when online cleanings occur in day 8,14 and 20. Note that the performance level of utility unit i2 declines in a slightly varied rate from day 17 to 18 and 24 to 25 due to its operating level deviation from its maximum capacity (see Figure 13). Recall unit performance levels are subject to terminal constraints (i.e., higher than $25 \%$ of the maximum performance of each unit). The performance level of utility unit $i 1$ in day 28 is below $25 \%$, but this is not a violation of the terminal constraint as already discuss before for the inventory level terminal constraints.

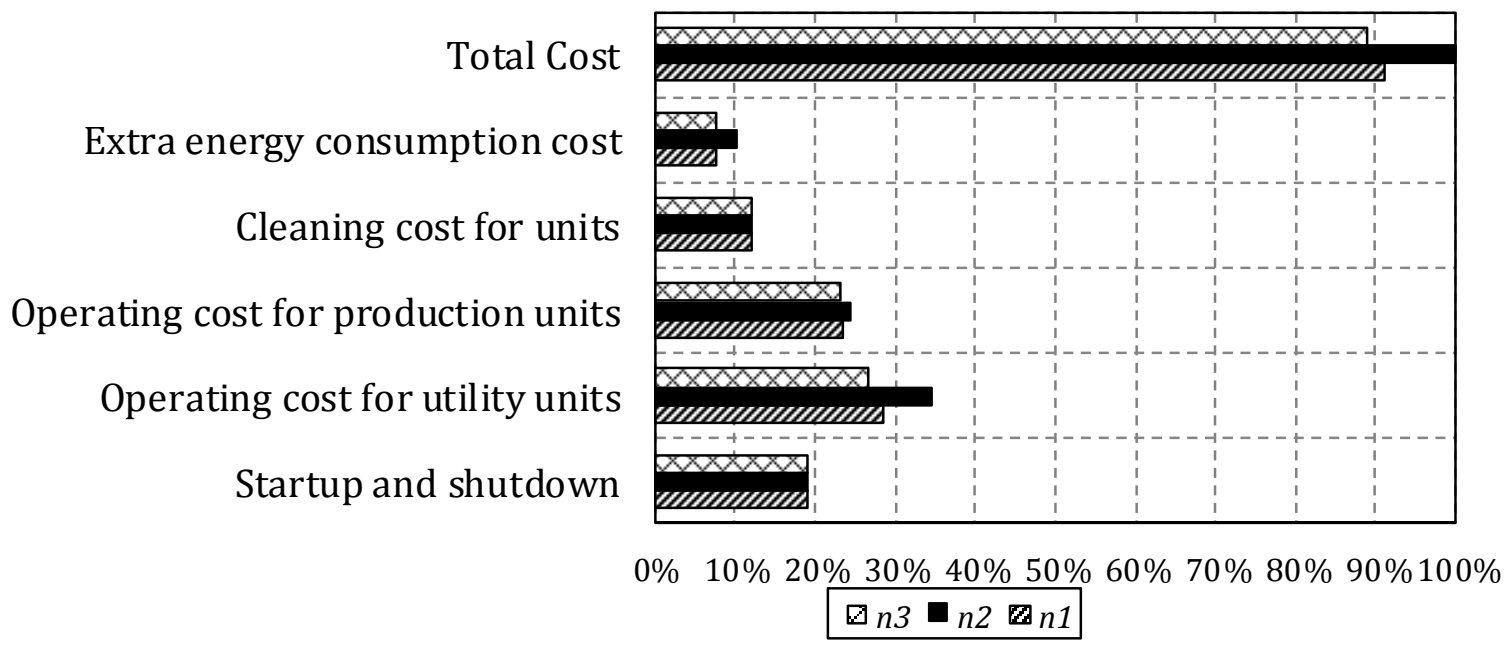

Figure 17: Case Study 2 - Rolling Horizon Stochastic Programming Approach: Cost term comparison for each scenario.

According to Figure 17 that shows a comparative cost breakdown among scenarios, total cost in high-demand scenario $n 2$ is $8.9 \%$ and $10.9 \%$ higher than that in medium-demand scenario $n 1$ and low-demand scenario $n 3$, respectively. Similarly to the previous case study, the major cost difference is observed in the operating cost for utility units in scenario $n 2$ which is $16.6 \%$ and $21.9 \%$ higher than that in scenario $n 1$ and scenario $n 3$, respectively. Extra energy consumption in scenario $n 2$ is $24.6 \%$ and $24.0 \%$ than that in scenario $n 1$ and $n 3$, respectively. Finally, the operating cost for production units in scenario $n 2$ is $2.7 \%$ and $3.7 \%$ higher than that in scenario $n 1$ and $n 3$, respectively. 


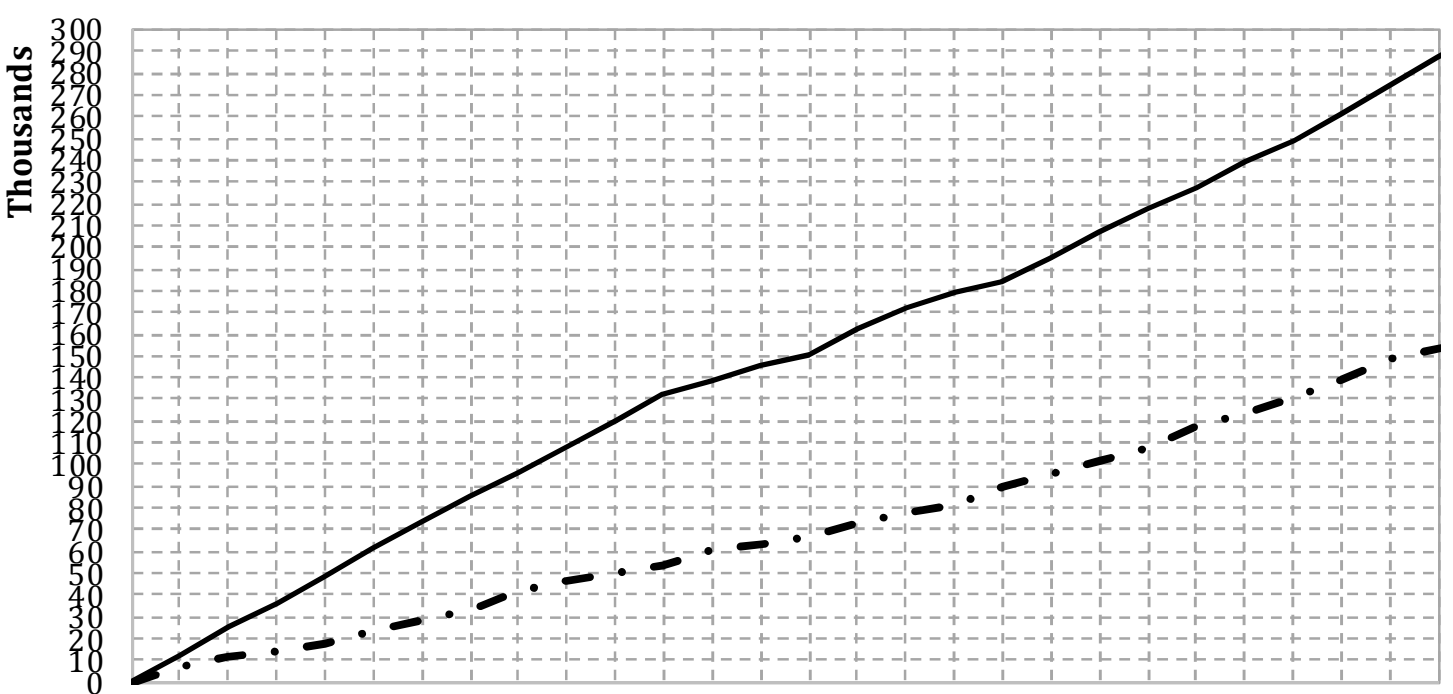

$1 \quad 2 \quad 3 \quad 4 \quad 5 \quad 6 \quad 7 \quad 8 \quad 9 \quad 10111213141516171819202122232425262728$

$$
\text { - Case Study } 2 \text { - - Perfect Information }
$$

\section{Figure 18: Case Study 2 - Rolling Horizon Stochastic Programming Approach: Aggregated total cost comparison.}

Figure 18 displays the aggregated total cost for rolling horizon stochastic programming approach and perfect information solution. The active scenario in the perfect information solution changes for every time period in the current prediction horizon. The results show that the total cost of the case study 2 is $48 \%$ higher than that of the perfect information solution. The perfect information solution is the best solution one could obtain. However, in practice this solution is impossible to be found due to uncertainty in the demand for products. It should be clear that the obtained solution could be improved, if the accuracy to forecast uncertainty is improved and the length of prediction horizon increases.

\subsection{Discussion on problem size and computational performance}

The size of the optimization models depend strongly on the number of time periods considered that affects directly the computational time of the resulting optimization problems. Table 3 shows how the computational time increases dramatically by increasing the number of time intervals, having as a reference Case Study 1 and considering 3 scenarios. However, it will be impossible to solve the integrated planning problem for a year planning horizon due to very hard optimization problem without the development of a decomposition method. In addition, the problem size will grow exponentially with increase number of scenarios because the model is getting bigger with respect to number of constraints and continuous variables, although the number of binary variables remains the same (for the same number of time periods). Notice that, the most appropriate method to solve stochastic problems with increase number of scenarios over multiple time periods is through multi-stage stochastic programming approach whereas the number of scenarios in the first time period increases exponentially with the length of total planning horizon considered. It has been also observed that the assigned scenario probabilities also affect the computational time. 
Table 3. Case Study 1: Computational results for different planning horizons.

\begin{tabular}{|l|l|l|l|l|}
\hline $\begin{array}{l}\text { Planning } \\
\text { Horizon }\end{array}$ & Equations & Continous Vars & Binary Vars & CPU s \\
\hline 7 days & 3,041 & 1,807 & 511 & 2 \\
\hline 14 days & 5,947 & 3,514 & 923 & 43,202 \\
\hline 21 days & 8,838 & 5,257 & 1,371 & 86,400 \\
\hline
\end{tabular}

\section{Conclusions}

A hybrid reactive/proactive optimization framework for the operational and resourceconstrained condition-based cleaning planning problem of integrated production and utility systems under uncertainty has been presented in this work. The proposed approach relies on a two-stage scenario-based stochastic programming model for the problem in question, applied within a rolling horizon scheme. Improved unit performance degradation and recovery models based on cumulative operating level deviations and cumulative operating times have been presented. Terminal constraints for minimum inventory levels for utilities and products as well as maximum unit performance degradation levels have been introduced too. Although in the case studies, demand uncertainty has been only considered, the proposed method can deal with several other types of uncertainty (e.g., price fluctuations). The proposed approach provides significant support to decision makers, since it can obtain the detailed optimal operational and cleaning plan of the utility and production system as a whole, and reporting operating levels profiles for units, performance level profiles for units, total production profiles for resources, inventory profiles, and total costs. The case studies presented highlighted the particular features and showed the applicability of the proposed approach as an effective means of dealing with the integrated planning problem considered under dynamic environments. Ongoing research activities focus on the modeling of network-based production systems with multiple stage production processes, mixing and splitting of materials and recycle flows. Problem decomposition methods are also studied for increasing the computational efficiency of the proposed approach.

\section{Acknowledgments}

The authors would like to express their gratitude to the Ministry of Higher Education Malaysia for providing financial support under the Scheme of Academic Training Reward (470/2015/41) throughout the realization of this research work.

\section{NOMENCLATURE}

Indices / Sets

$\begin{array}{ll}e \in E & \text { resources (products and utilities) } \\ i \in I & \text { units (production and utility) } \\ n \in N & \text { scenarios } \\ q \in Q & \text { offline cleaning task options } \\ t \in T & \text { time periods } \\ z \in Z & \text { inventory tanks for resources }\end{array}$

\section{Superscripts}

es earliest

Is latest

max maximum 


$\begin{array}{ll}\text { min } & \text { minimum } \\ \text { off } & \text { offline } \\ \text { on } & \text { online } \\ \text { fix } & \text { fixed } \\ \text { var } & \text { variable } \\ \text { PR } & \text { production system } \\ \text { UT } & \text { utility system } \\ + & \text { inlet } \\ - & \text { outlet }\end{array}$

\section{Subsets}

$E_{i}$

resources that can be produced in unit $i$

$E^{P R}$

$E^{U T}$

product resources

$I_{e}$

utility resources

$I^{S F}$

$I^{S-\min }$

units that can produced resource $e$

units that are subject to startup and shutdown costs

$I^{F-\min }$

units that are subject to minimum runtimes

$I_{e}^{P R}$

units that are subject to minimum shutdown times

$Q_{i}$

production units that require utility $e$ to operate

alternative offline cleaning task options for unit $i$

$Z_{e}$

$Z_{i}$

$C B_{i}$

inventory tanks that can store resource $e$

units $i$ connected to the input/output of inventory tank $z$

$D M_{i}$ units $i$ that are subject to condition-based cleaning tasks

units $i$ that are under in-progress offline cleaning at the beginning of the planning horizon (information carried over from previous planning horizon)

$F M_{i} \quad$ units $i$ that are subject to flexible time-window offline cleaning

$M R_{i} \quad$ units $i$ that are subject to maximum runtime constraints

$P R_{i} \quad$ production units

$U T_{i} \quad$ utility units

\section{Parameters}

$\alpha_{\left(i, e, e^{\prime}\right)}$

coefficient for production unit $i$ that provides the variable needs for utility $e$ for the production of a unit of product $e^{\prime}$

$\bar{\alpha}_{\left(i, e, e^{\prime}\right)}$ coefficient for production unit $i$ that provides the fixed needs for utilities $e$ for the production of product $e^{\prime}$

$\beta_{z}^{\text {loss }} \quad$ coefficient of losses in inventory tank

$\gamma_{i}^{o n}$

minimum time between two consecutive online cleanings in unit $i$

$\delta_{i}$ performance degradation rate for unit $i$ due to its cumulative time of operation 


\begin{tabular}{|c|c|}
\hline$\delta_{i}^{q}$ & performance coefficient related to operating level for unit $i$ due to its \\
\hline$\delta_{n}^{p}$ & probability of occurrence for each scenario $n$ \\
\hline$\varepsilon_{(e, z, t)}$ & $\begin{array}{l}\text { bounds on the total inlet/outlet flow of resource } e \text { to/from inventory tank } z \text { in } \\
\text { time period } t\end{array}$ \\
\hline$\zeta_{(e, \mathrm{t})}$ & demand for product $e$ in time period $t$ \\
\hline$\eta_{t}^{\max }$ & limited amount of available resources for cleaning operations in time period $t$ \\
\hline$\vartheta_{(i, q)}^{o f f}$ & resource requirements for offline cleaning task option $q$ of unit $i$ \\
\hline$\vartheta_{i}^{o n}$ & resource requirements for online cleaning of unit $i$ \\
\hline$\kappa_{(i, t)}$ & bounds on the operating level for utility unit $i \in U T_{i}$ in time period $t$ \\
\hline $\bar{\kappa}_{(i, e, t)}$ & $\begin{array}{l}\text { bounds on the production level of product } e \text { for production unit } i \in P R_{i} \text { in time } \\
\text { period } t\end{array}$ \\
\hline$\lambda_{(e, z)}^{B}$ & $\begin{array}{l}\text { percentage coefficient that determines the minimum level for each resource } \\
\text { inventory tank at the end of the prediction horizon (terminal value) }\end{array}$ \\
\hline$\lambda_{i}^{U}$ & $\begin{array}{l}\text { percentage coefficient that determines the maximum performance degradation } \\
\text { level for operating unit } i \text { at the end of the prediction horizon (terminal value) }\end{array}$ \\
\hline$\mu_{(i, t)}, \bar{\mu}_{(i, t)}$ & sufficient big numbers \\
\hline$v_{(i, q)}$ & duration of offline cleaning task option $q$ that could take place in unit $i$ \\
\hline$\xi_{(e, z)}$ & bounds on the capacity of inventory tanks $z$ that can store resources $e$ \\
\hline$o_{i}$ & maximum runtime for unit $i$ \\
\hline$\pi_{i}^{n}$ & $\begin{array}{l}\text { percentage modification on the upper operating level of unit } i \text { that is under } \\
\text { online cleaning }\end{array}$ \\
\hline$\rho_{(i, e)}$ & $\begin{array}{l}\text { stoichiometry coefficient that relates the operating level of the utility unit } i \text { with } \\
\text { the generated amount of each cogenerated utility } e\end{array}$ \\
\hline$\rho_{i}^{r e c}$ & recovery factor for unit $i$ due to online cleaning (partial performance recovery) \\
\hline$\tau_{i}$ & time information of offline cleaning tasks for unit $i$ \\
\hline$v_{i}^{\max }$ & extra energy consumption limit for unit $i$ (performance degradation) \\
\hline$\phi$ & $\begin{array}{l}\text { associated cost coefficients for objective function terms related to utility and } \\
\text { production unit } i \text { (i.e., variable and fixed operating cost, utilities and products } \\
\text { purchase prices, startup and shutdown costs, extra energy consumption cost, } \\
\text { online and offline cleaning tasks costs) }\end{array}$ \\
\hline$\psi_{i}$ & minimum shutdown idle time for unit $i$ \\
\hline (1) & minimum run \\
\hline
\end{tabular}


$q_{(i, t)}^{r e f}$
$q_{(i, e, t)}^{r e f}$

reference operating level for utility unit $i$ per time period $t$

reference production level for production unit $i$ that produces product $e$ per time period $t$

\section{Parameters (initial state of the overall system)}

$\tilde{\beta}_{(e, z)}$

$\tilde{\gamma}_{i}^{n n}$

$\tilde{\eta}_{(i, t)}$

$\tilde{\rho}_{i}$

$\tilde{\rho}_{i}^{q}$

$\tilde{\chi}_{i}$

$\tilde{\psi}_{i}$

$\tilde{\omega}_{i}$

initial inventory level of resource $e$ in inventory tank $z$ initial state of utility unit $i \in C B_{i}^{o n}$ with respect to its last online cleaning time periods $t$ for utility unit $i \in D M_{i}$ that there is a known cleaning resource requirement (in-progress offline cleaning task from previous planning horizon) initial cumulative time of operation for unit $i$ initial cumulative deviation from the reference operating level for unit $i$ operating status of unit $i$ just before the start of the current planning horizon total number of time periods at the beginning of the current planning horizon that unit $i$ has been continuously not operating since its last shutdown total number of time periods at the beginning of the current planning horizon that unit $i$ has been continuously operating since its last startup

\section{Continuous variables (non-negative)}

$B_{(n, e, z, t)}$

$B_{(n, e, z, t)}^{-}$

$B_{(n, e, z, t)}^{+}$

$B_{(n, e, z, i, t)}^{U T,-}$

$D_{(n, i, t)}$

$N S_{(n, e, i, t)}^{U T}$

$N S_{(n, e, t)}^{F P}$

$\bar{Q}_{(n, i, t)}$

$Q_{(n, i, e, t)}$

$Q_{(n, i, e, t)}^{d e v}$

$\bar{Q}_{(n, i, t)}^{d e v}$

$R_{(i, t)}$ inventory level for resource $e$ in inventory tank $z$ at time $t$ for scenario $n$ total outlet flow of resource $e$ from inventory tank $z$ at time period $t$ for scenario $n$

total inlet flow of resource $e$ to inventory tank $z$ at time period $t$ for scenario $n$

flow of utility $e$ from inventory tank $z$ to production unit $i$ at time period $t$ for scenario $n$

cumulative operating level deviation for unit $i$ in time period $t$ for scenario $n$ purchases of utility $e$ to be utilized in production unit $i \in I_{e}^{P R}$ in time period $t$ for scenario $n$

purchases of product $e$ in time period $t$ (or lost sales) for scenario $n$ operating level of utility unit $i$ in time period $t$ for scenario $n$ production level of resource $e$ from unit $i$ in time period $t$ for scenario $n$ operating level deviation of the production unit $i$ from its reference operating level in time period $t$ for scenario $n$ operating level deviation of the utility unit $i$ from its reference operating level in time period $t$ for scenario $n$ cumulative time of operation for unit $i$ in time period $t$ 
$U_{(n, i, t)} \quad$ extra energy consumption (from fully clean condition) of unit $i$ due to its performance degradation for scenario $n$

\section{Binary variables}

$\begin{array}{ll}X_{(i, t)} & =1 \text {, if a unit } i \text { is operating during time period } t \\ S_{(i, t)} & =1 \text {, if a unit } i \text { starts up at the beginning of time period } t \\ F_{(i, t)} & =1 \text {, if a unit } i \text { shuts down at the beginning of time period } t \\ V_{(i, t)} & =1 \text {, if an online cleaning task for unit } i \in C B_{i}^{o n} \text { occurs in time period } t \\ V_{(i, e, t)}^{P R} & =1 \text {, if an online cleaning task for production unit } i \in\left(P R_{i} \cap C B_{i}^{o n}\right) \text { that produces } \\ & \text { product } e \in E^{P R} \text { takes place in time period } t \\ W_{(i, t)} & =1 \text {, if an offline cleaning task for unit } i \in\left(C B_{i}^{\text {off }} \cup F M_{i}\right) \text { starts at the beginning } \\ H_{(i, q, t)} & \quad \text { of time period } t \\ Y_{(i, e, t)} & \quad 1, \text { if the offline cleaning task option } q \in Q_{i} \text { for unit } i \in\left(C B_{i}^{\text {off }} \cup F M_{i}\right) \text { starts at } \\ & \text { the beginning of time period } t\end{array}$

\section{References}

Biyanto, T.R., Ramasamy, M., Jameran, A.B., Fibrianto, H.Y., 2016. Thermal and hydraulic impacts consideration in refinery crude preheat train cleaning scheduling using recent stochastic optimization methods. Applied Thermal Engineering 108, 1436-1450.

Choi, G.B., Lee, S.G., Lee, J.M., 2016. Multi-period energy planning model under uncertainty in market prices and demands of energy resources: A case study of Korea power system. Chemical Engineering Research and Design 114, 341-358.

Cobuloglu, H.I., Esra Büyüktahtakın, I., 2017. A two-stage stochastic mixed-integer programming approach to the competition of biofuel and food production. Computers and Industrial Engineering 107, 251-263.

Christofides, P.D, Davies, J.F., El-Farra, N.H., Clark, D., Harris, K.R.D., Gipson, J.N., 2007. Smart plant operations: vision, progress and challenges. AIChE Journal 53, 2734-2741.

Diaby, A.L., Miklavcic, S.J., Addai-Mensah, J., 2016. Optimization of scheduled cleaning of fouled heat exchanger network under ageing using genetic algorithm. Chemical Engineering Research and Design 113, 223-240.

Ekin, T., 2017. Integrated maintenance and production planning with endogenous uncertain yield. Reliability Engineering and System Safety 0, 1-10.

Gössinger, R., Helmke, H., Kaluzny, M., 2017. Condition-based release of maintenance jobs in a decentralised production-maintenance system - An analysis of alternative stochastic approaches. International Journal of Production Economics 193, 528-537.

Gupta, A., Maranas, C.D., 2000. A two-stage modeling and solution framework for multisite midterm planning under demand uncertainty. Industrial and Engineering Chemistry Research 39, 3799-3813.

Huang, Y.H., Wu, J.H., Hsu, Y.J., 2016. Two-stage stochastic programming model for the regional-scale electricity planning under demand uncertainty. Energy 116, 1145-1157.

Khatab, A., Aghezzaf, E.H., Djelloul, I., Sari, Z., 2017. Selective maintenance optimization for systems operating missions and scheduled breaks with stochastic durations. Journal of 
Manufacturing Systems 43, 168-177.

Kostin, A.M., Guillén-Gosálbez, G., Mele, F.D., Bagajewicz, M.J., Jiménez, L., 2012. Design and planning of infrastructures for bioethanol and sugar production under demand uncertainty. Chemical Engineering Research and Design 90, 359-376.

Samuelson, A., Haigh, A., O'Reilly, M.M., Bean, N.G., 2017. Stochastic model for maintenance in continuously deteriorating systems. European Journal of Operational Research 259, 1169-1179.

Silvente, J., Kopanos, G.M., Pistikopoulos, E.N., Espuña, A., 2015. A rolling horizon optimization framework for the simultaneous energy supply and demand planning in microgrids. Applied Energy 155, 485-501.

Silvente, J., Kopanos, G.M., Dua, V., Papageorgiou, L.G., 2017. A rolling horizon approach for optimal management of microgrids under stochastic uncertainty. Chemical Engineering Research and Design 1-25.

Zhou, Y., Lin, T.R., Sun, Y., Ma, L., 2016. Maintenance optimisation of a parallel-series system with stochastic and economic dependence under limited maintenance capacity. Reliability Engineering and System Safety 155, 137-146.

Zulkafli, N.I., Kopanos, G.M., 2016. Planning of production and utility systems under unit performance degradation and alternative resource-constrained cleaning policies. Applied Energy 183, 577-602.

Zulkafli, N.I., Kopanos, G.M., 2017. Integrated condition-based planning of production and utility systems under uncertainty. Journal of Cleaner Production 167, 776-805. 


\section{SUPPLEMENTARY MATERIAL}

\section{SM1. Startup and Shutdown Actions.}

The operational status of each unit is then modeled according to:

$$
\begin{array}{ll}
S_{(i, t)}-F_{(i, t)}=X_{(i, t)}-\tilde{\chi}_{i} & \forall i \in I^{S F}, t \in T: t=1 \\
S_{(i, t)}-F_{(i, t)}=X_{(i, t)}-X_{(i, t-1)} & \forall i \in I^{S F}, t \in T: t>1 \\
S_{(i, t)}+F_{(i, t)} \leq 1 & \forall i \in I^{S F}, t \in T
\end{array}
$$

The minimum runtime $\left(\omega_{i}\right)$ and shutdown time $\left(\psi_{i}\right)$ for any unit subject to minimum runtime or shutdown restriction are modeled by constraints (1-2) and (1-3), respectively.

$$
\begin{aligned}
& X_{(i, t)} \geq \sum_{t^{\prime}=\max \left\{1, t-\omega_{i}+1\right\}}^{t} S_{\left(i, t^{\prime}\right)} \quad \forall i \in I^{S-m i n}, t \in T: \omega_{i}>1 \\
& X_{(i, t)}=1 \\
& 1-X_{(i, t)} \geq \sum_{t^{\prime}=\max \left\{1, t-\psi_{i}+1\right\}}^{t} F_{\left(i, t^{\prime}\right)} \quad \forall i \in I^{S-\min }, t=1, \ldots,\left(\omega_{i}-\tilde{\omega}_{i}\right): 0<\tilde{\omega}_{i}<\omega_{i} \\
& X_{(i, t)}=0 \quad \forall i \in I^{F-\min }, t=1, \ldots,\left(\psi_{i}-\tilde{\psi}_{i}\right): 0<\tilde{\psi}_{i}<\psi_{i}
\end{aligned}
$$

A maximum runtime $\left(o_{i}\right)$ may be imposed for units $i \in M R_{i}$ that do not follow a more detailed performance-based cleaning planning, according to:

$$
\begin{array}{ll}
\sum_{t^{\prime}=\max \left\{1, t-o_{i}\right\}}^{t} X_{\left(i, t^{\prime}\right)} \leq o_{i} & \forall i \in M R_{i}, t \in T \\
\sum_{t^{\prime}=\max \left\{1, t-\left(o_{i}-\tilde{\omega}_{i}\right)\right\}}^{t} X_{\left(i, t^{\prime}\right)} \leq\left(o_{i}-\tilde{\omega}_{i}\right) & \forall i \in M R_{i}, t=\left(o_{i}-\tilde{\omega}_{i}+1\right): \tilde{\omega}>1
\end{array}
$$

\section{SM2. Cleaning Tasks.}

\section{SM2.1 In-progress offline cleaning tasks.}

At the beginning of the planning horizon, there may be some in-progress unfinished offline cleaning tasks for some units $\left(i \in D M_{i}\right)$ which are carried over from the previous planning horizon. These cleaning tasks are modeled according to:

$$
X_{(i, t)}=0 \quad \forall i \in D M_{i}, t \in T: \tilde{\eta}_{(i, t)}>0
$$

\section{SM2.2 Flexible time-window offline cleaning tasks.}

There may be alternative options for these offline cleaning tasks. And as such, one cleaning task option need to start within the given time window $t=\left[\tau_{i}^{e s}, \tau_{i}^{l s}\right]$, as given by: 
$\sum_{q \in Q_{i}} \sum_{t=\tau_{i}^{\tau_{i}^{s}}}^{\tau_{i s}^{l s}} H_{(i, q, t)}=1 \quad \forall i \in F M_{i}$

\section{SM2.3 Condition-based online cleaning tasks.}

In any given time period, a unit could be under online cleaning only if the unit is under operation during this period, as modeled by:

$V_{(i, t)} \leq X_{(i, t)} \quad \forall i \in C B_{i}^{o n}, t \in T$

A unit can undergo an online cleaning task after a minimum time period has passed from the occurrence of the previous online cleaning task in the same unit, as given by:

$\begin{array}{ll}\sum_{t^{\prime}=\max \left\{1, t-\gamma_{i}^{o n}+1\right\}}^{t} V_{\left(i, t^{\prime}\right)} \leq 1 & \forall i \in C B_{i}^{o n}, t \in T \\ V_{(i, t)}=0 & \forall i \in C B_{i}^{o n}, t \leq\left(\gamma_{i}^{o n}-\tilde{\gamma}_{i}^{o n}\right): \tilde{\gamma}_{i}^{o n}<\gamma_{i}^{o n}\end{array}$

Constraints (2-5) relate the two binary variables for online cleaning tasks for the production units. These constraints are needed in order to model correctly the modified maximum operating levels of production units during the period that are under online cleaning.

$V_{(i, t)}=\sum_{e \in E_{i}} V_{(i, e, t)}^{P R} \quad \forall i \in\left(C B_{i}^{o n} \cap P R_{i}\right), t \in T$

SM2.4 Operational constraints for offline cleaning tasks.

Constraints (2-6) and (2-7) ensure that a unit that is under offline cleaning remains closed for the whole duration of the selected offline cleaning task option, and relate the two binary variables for offline cleaning tasks.

$$
\begin{aligned}
& X_{(i, t)}+\sum_{t^{\prime}=\max \left\{\tau_{i}^{e s}, t-v_{(i, q)}+1\right\}}^{\min \left\{\tau_{i}^{l s}, t\right\}} H_{\left(i, q, t^{\prime}\right)} \leq 1 \quad \forall i \in\left(F M_{i} \cup C B_{i}^{\text {off }}\right), q \in Q_{i}, \tau_{i}^{e s} \leq t \leq\left(\tau_{i}^{l s}+v_{(i, q)}-1\right) \\
& W_{(i, t)}=\sum_{q \in Q_{i}} H_{(i, q, t)} \quad \forall i \in\left(F M_{i} \cup C B_{i}^{o f f}\right), t \in T: \tau_{i}^{e s} \leq t \leq \tau_{i}^{l s}
\end{aligned}
$$

For condition-based offline cleaning tasks, earliest and latest starting times should be set equal to the first and the last period of the planning horizon, respectively.

\section{SM2.5 Resource constraints for cleaning tasks.}

A limited amount of available resources for cleaning operations shared by all types of cleaning tasks is considered, according to:

$$
\begin{aligned}
\sum_{i \in C B_{i}^{o n}} \vartheta_{i}^{o n} V_{(i, t)}+\sum_{i \in C B_{i}^{o f f}} \sum_{q \in Q_{i} t^{\prime}=t-v_{(i, q)}+1} \sum_{(i, q)}^{t} H_{\left(i, q, t^{\prime}\right)}^{\text {off }}+\sum_{i \in F M_{i}} \sum_{q \in Q_{i} t^{\prime}=\max \left\{\left\{\tau_{i}^{e s}, t-v_{(i, q)}+1\right\}\right.} \sum_{\left(i, q, t^{\prime}\right)}^{\min \left\{\tau_{i}^{l s}, t\right\}} & \leq \eta_{t}^{\max }-\sum_{i \in D M_{i}} \tilde{\eta}_{(i, t)} \quad \forall t \in T
\end{aligned}
$$

